\title{
On some Hamiltonian properties of the isomonodromic tau functions
}

\author{
A. R. Its 1, A. Prokhorol? \\ Department of Mathematical Sciences, Indiana University-Purdue University, 402 N. Blackford St., \\ Indianapolis, IN 46202-3267, USA.
}

St. Petersburg State University, Universitetskaya emb. 7/9, 199034, St. Petersburg, Russia.

\begin{abstract}
We discuss some new aspects of the theory of the Jimbo-Miwa-Ueno tau function which have come to light within the recent developments in the global asymptotic analysis of the tau functions related to the Painlevé equations. Specifically, we show that up to the total differentials the logarithmic derivatives of the Painlevé tau functions coincide with the corresponding classical action differential. This fact simplifies considerably the evaluation of the constant factors in the asymptotics of tau-functions, which has been a long-standing problem of the asymptotic theory of Painlevé equations. Furthermore, we believe that this observation is yet another manifestation of L. D. Faddeev's emphasis of the key role which the Hamiltonian aspects play in the theory of integrable system.
\end{abstract}

This article will appear in the WSPC memorial volume dedicated to Ludwig Faddeev.

\section{Introduction}

Consider a system of linear ordinary differential equations with rational coefficients,

$$
\frac{d \Phi}{d z}=A(z) \Phi
$$

where $A(z)$ is an $N \times N, N>1$ matrix-valued rational function, The object of our study is the Jimbo-Miwa-Ueno tau function associated with the isomonodromic deformation of system (1). Let us remind, following [JMU], the general set-up associated with this notion.

Denote the poles of the matrix valued rational function $A(z)$ on $\mathbb{C} P^{1}$ by $a_{1}, \ldots, a_{n}, \infty$ and by $r_{1}, r_{2}, \ldots, r_{n}, r_{\infty}$ the corresponding Poincaré ranks. The matrix function $A(z)$ can be then written as,

$$
A(z)=\sum_{v=1}^{n} \sum_{k=1}^{r_{v}+1} \frac{A_{v,-k+1}}{\left(z-a_{v}\right)^{k}}+\sum_{k=0}^{r_{\infty}-1} z^{k} A_{\infty,-k-1}, \quad A_{v,-k+1}, A_{\infty,-k-1} \in \mathfrak{s l}_{N}(\mathbb{C}), \quad k=1, \ldots, r_{v}+1, \quad v=1, \ldots, n
$$

We are going to make the standard assumption that all highest order matrix coefficients $A_{v} \equiv A_{v,-}$ are diagonalizable

$$
A_{v,-r_{v}}=G_{v} \Theta_{v,-r_{v}} G_{v}^{-1} ; \quad \Theta_{v,-r_{v}}=\operatorname{diag}\left\{\theta_{v, 1}, \ldots \theta_{v, N}\right\},
$$

and that their eigenvalues are distinct and non-resonant:

$$
\begin{cases}\theta_{v, \alpha} \neq \theta_{v, \beta} & \text { if } \quad r_{v} \geq 1, \quad \alpha \neq \beta, \\ \theta_{v, \alpha} \neq \theta_{v, \beta} \quad \bmod \mathbb{Z} \quad \text { if } \quad r_{v}=0, \quad \alpha \neq \beta .\end{cases}
$$

At each singular point, the system (1) admits a unique formal solution,

$$
\Phi_{\text {form }}^{(v)}(z)=G^{(v)}(z) e^{\Theta_{v}(z)}, \quad v=1, \ldots, n, \infty,
$$

\footnotetext{
${ }^{1}$ aits@iupui.edu

${ }^{2}$ aprokhor@iupui.edu
} 
where $G^{(v)}(z)$ are formal series,

$$
G^{(v)}(z)=G_{v}\left[I+\sum_{k=1}^{\infty} g_{v, k}\left(z-a_{v}\right)^{k}\right], \quad G^{(\infty)}(z)=G_{\infty}\left[I+\sum_{k=1}^{\infty} g_{\infty, k} z^{k}\right],
$$

and $\Theta_{v}(z)$ are diagonal matrix-valued functions,

$$
\Theta_{v}(z)=\sum_{k=-r_{v}}^{-1} \frac{\Theta_{v, k}}{k}\left(z-a_{v}\right)^{k}+\Theta_{v, 0} \ln \left(z-a_{v}\right), \quad \Theta_{\infty}(z)=-\sum_{k=1}^{r_{\infty}} \frac{\Theta_{\infty,-k}}{k} z^{k}-\Theta_{\infty, 0} \ln z .
$$

For every $v \in\{1, \ldots, n, \infty\}$, the matrix coefficients $g_{v, k}$ and $\Theta_{v, k}$ can be explicitly computed in terms of the coefficients of the matrix-valued rational function $G_{v}^{-1} A(z) G_{v}$, see [JMU].

The non-formal global properties of solutions of the equation (1) are described by its monodromy data $M$ which include: i) formal monodromy exponents $\Theta_{v, 0}$, ii) appropriate connection matrices between canonical solutions at different singular points, and iii) relevant Stokes matrices at irregular $\left(r_{v} \geq 1\right)$ singular points. Let us denote the space of monodromy data of the system (1) by $\mathscr{M}$. It can be described in more details as follows.

Let $a_{v}$ be an irregular singular point of index $r_{v}$. For $j=1, \ldots, 2 r_{v}+1$, let also

$$
\Omega_{j, v}=\left\{z: 0<\left|z-a_{v}\right|<\epsilon, \quad \theta_{j}^{(1)}<\arg \left(z-a_{v}\right)<\theta_{j}^{(2)}, \quad \theta_{j}^{(2)}-\theta_{j}^{(1)}=\frac{\pi}{r_{v}}+\delta\right\}
$$

be the Stokes sectors around $a_{v}$ (see, e.g., [FIKN], Chapter 1] or [Was for more details). According to the general theory of linear systems, in each sector $\Omega_{j, v}$ there exists a unique canonical solution $\Phi_{j}^{(v)}(z)$ of 1 which satisfies the asymptotic condition

$$
\Phi_{j}^{(v)}(z) \simeq \Phi_{\text {form }}^{(v)}(z) \quad \text { as } z \rightarrow a_{v}, \quad z \in \Omega_{j, v}, \quad j=1, \ldots, 2 r_{v}+1 .
$$

Different canonical solutions are related by Stokes matrices, $S_{j}^{(v)}$, and connection matrices, $C_{v}$ :

$$
\Phi_{j+1}^{(v)}=\Phi_{j}^{(v)} S_{j}^{(v)}, \quad j=1, \ldots, 2 r_{v}, \quad \Phi_{1}^{(v)}=\Phi_{1}^{(\infty)} C_{v}, \quad v=1, \ldots, n .
$$

Let us assume that the irregular singular points are $\infty$ and the first $n_{0} \leq n$ points among the singular points $a_{1}, \ldots, a_{n}$. Denote by $\mathscr{S}_{v}$ the collection of Stokes matrices at an irregular point $a_{v}$, i.e.

$$
\mathscr{S}_{v}=\left\{S_{1}^{(v)}, \ldots, S_{2 r_{v}}^{(v)}\right\} .
$$

The space $\mathscr{M}$ of monodromy data of the system (1) consists of formal monodromy exponents $\Theta_{v, 0}$, connection matrices $C_{v}$ and Stokes matrices $S_{j}^{(v)}$, i.e.,

$$
\mathscr{M}=\left\{M \equiv\left(\Theta_{v, 0}, v=1, \ldots, n, \infty ; \quad C_{v}, v=1, \ldots, n ; \quad \mathscr{S}_{v}, v=1, \ldots, n_{0}, \infty\right)\right\} .
$$

We shall use the notation,

$$
\vec{m}=\left(m_{1}, \ldots, m_{d}\right), \quad d=N(n+1)+n N^{2}+\left(\frac{N(N-1)}{2}\right)\left(\sum_{v=1}^{n_{0}} 2 r_{v}+2 r_{\infty}\right),
$$

for the points $\vec{m} \in \mathscr{M}$. In addition, we denote by $\mathscr{T}$ the set of times,

$$
a_{1}, \ldots, a_{n}, \quad\left(\Theta_{v, k}\right)_{l l}, \quad k=-r_{v}, \ldots,-1, \quad v=1, \ldots, n_{0}, \infty, \quad l=1, \ldots, N .
$$

We shall use the notation,

$$
\vec{t}=\left(t_{1}, \ldots, t_{L}\right), \quad L=n+N\left(\sum_{v=1}^{n_{0}} r_{v}+r_{\infty}\right),
$$

for the points $\vec{t} \in \mathscr{T}$. Let us also denote by $\mathscr{A}$ the variety of all rational matrix-valued functions $A(z)$ with a fixed number of poles of fixed orders. The so-called Riemann-Hilbert correspondence states that, up to submanifolds where the inverse monodromy problem for (1) is not solvable, the space $\mathscr{A}$ can be identified with the product $\widetilde{\mathscr{T}} \times \mathscr{M}$, where $\widetilde{\mathscr{T}}$ denotes the universal covering of $\mathscr{T}$. We shall loosely write,

$$
\mathscr{A} \simeq \widetilde{\mathscr{T}} \times \mathscr{M}
$$


It should be mentioned that in each concrete case one has to specify the gauge normalization of the matrix $A(z)$ as well as the choice of the gauge matrices $G_{v}$ in order to make this identification well defined. In Section 3 we will demonstrate how these specifications can be done in the case of Painlevé equations.

The Jimbo-Miwa-Ueno 1-form is defined as the following differential form on $\mathscr{A}$ :

$$
\omega_{\mathrm{JMU}}=-\sum_{v=1, \ldots, n, \infty} \underset{z=a_{v}}{\operatorname{res}} \operatorname{Tr}\left(\left(G^{(v)}(z)\right)^{-1} \frac{d G^{(v)}}{d z}(z) d_{\mathscr{T}} \Theta_{v}(z)\right),
$$

where $G^{(v)}(z)$ are the series from [2] and we put $a_{\infty} \equiv \infty$. The notation $d_{\mathscr{T}} \Theta_{v}(z)$ stands for

$$
d \Theta_{v}(z)=\sum_{k=1}^{L} \frac{\partial \Theta_{v}(z)}{\partial t_{k}} d t_{k}, \quad L=n+N\left(\sum_{v=1}^{n_{0}} r_{v}+r_{\infty}\right) .
$$

The significance of this form is that, being restricted to any isomonodromic family in the space $\mathscr{A}$,

$$
A(z) \equiv A(z ; \vec{t}, M), \quad \vec{t}=\left(t_{1}, \ldots, t_{L}\right), \quad M \equiv \mathrm{const}
$$

it becomes closed with respect to times $\mathscr{T}$, i.e.

$$
d_{\mathscr{T}}\left(\left.\omega_{\mathrm{JMU}}\right|_{A(z ; \vec{t}, M \equiv \text { const })}\right)=0 .
$$

The closedness of the 1 -form $\omega_{\text {JMU }}$ with respect to $\mathscr{T}$ in turn implies that locally there is a function $\tau \equiv \tau(\vec{t} ; M)$ on $\mathscr{T} \times \mathscr{M}$ such that

$$
d_{\mathscr{T}} \ln \tau=\left.\omega_{\mathrm{JMU}}\right|_{A(z ; \vec{t}, M)} .
$$

A remarkable property of the tau function $\tau(\vec{t}, M)$, which was established in $[$ Mal] and [Miw], is that it admits analytic continuation as an entire function to the whole universal covering $\widetilde{\mathscr{T}}$ of the parameter space $\mathscr{T}$. Furthermore, zeros of $\tau(\vec{t}, M)$ correspond to the points in $\mathscr{T}$ where the inverse monodromy problem for (1) is not solvable for a given set $M$ of monodromy data (or, equivalently, where the holomorphic vector bundle over $\mathbb{C} P^{1}$ determined by $M$ becomes nontrivial). Hence a central role of the concept of tau function in the monodromy theory of systems of linear differential equations.

The isomonodromicity of the family $A(z ; \vec{t}, M)$ means that all equations from it have the same set $M \in \mathscr{M}$ of monodromy data. This implies that the corresponding solution $\Phi(z) \equiv \Phi(z, \vec{t})$ satisfies an overdetermined system

$$
\left\{\begin{array}{l}
\partial_{z} \Phi=A(z, \vec{t}) \Phi(z, \vec{t}) \\
d_{\mathscr{T}} \Phi=B(z, \vec{t}) \Phi(z, \vec{t})
\end{array} .\right.
$$

The coefficients of the matrix-valued differential form $B \equiv \sum_{k=1}^{L} B_{k}(z, \vec{t}) d t_{k}$ are rational in $z$. Their explicit form may be algorithmically deduced from the expression for $A(z)$ (see again [JMU]). The compatibility of the system (5) yields the monodromy preserving deformation equation:

$$
d_{\mathscr{T}} A=\partial_{z} B+[B, A] .
$$

Isomonodromy equation [6] is of great interest on its own. Indeed, it includes as special cases practically all known integrable differential equations. The first nontrivial cases of [6], where the set of isomonodromic times effectively reduces to a single variable $t$, cover all six classical Painlevé equations. Solutions of the latter are dubbed as nonlinear special functions, and they indeed play this role in many areas of modern nonlinear science (see [FIKN], [BK], [DS], [GM], [TW1], [TW2]).

The principal analytic issue concerning the tau function, in particular from the point of view of applications, is its behavior near the critical hyperplanes, where either $a_{\mu}=a_{v}$ for some $\mu \neq v$, or $\theta_{v, \alpha}=\theta_{v, \beta}$ for some $v$ and some $\alpha \neq \beta$. In the case of Painlevé equations this is the behavior of respective tau functions near the $t=\infty$ (PI, II, IV), $t=\infty, 0$ (PIII, V), and $t=\infty, 0,1$ (PVI). A special challenge in the asymptotic analysis of the tau functions is the evaluation of the constant pre-factors in their asymptotics. In fact, it is these pre-factors which usually contain the most important information about the physical properties of the model under investigation. At the same time, they can not be obtained directly via the Riemann-Hilbert approach. The latter method is one of the principal modern tools of the asymptotic analysis of Painleve transcendents, and it is 
based on the asymptotic evaluation of the above mentioned Riemann-Hilbert correspondence. In other words, the Riemann-Hilbert technique allows to evaluate the asymptotics of the matrix $A(z, \vec{t})$ and hence the asymptotics of the differential form $\omega_{\text {JMU. }}$. In view of 4, this gives the asymptotics of the logarithmic derivatives of the tau function. In order to obtain the complete asymptotic description of the tau function itself, which would include the above mentioned pre-factors, one has to solve the "constant problem": to find the constant of integration arising from the formal integration of (4). More precisely, since the tau function is itself defined up to a multiplicative constant, we are actually talking about the evaluation, in terms of monodromy data, of the ratios of constant factors corresponding to different critical points (Painlevé III, V, VI) or to different critical directions (Painlevé I, II, IV).

The first rigorous solution of a constant problem for Painlevé equations (a special Painlevé III transcendent appearing in the Ising model) has been obtained in the work of C. Tracy [Tr]. After that, several other important special cases have been also solved. We refer the reader to ILP for a detailed history of the question. It is important to emphasize that all these works were concerned with the very special families of the Painlevé functions, and they used the techniques which could not be extended to the generic tau functions.

The means to solve the "constant problem" for tau functions corresponding to the generic solutions of Painlevé equations started to develop since the 2013-2014 works [ILT13, [ILST] of Iorgov, Lisovyy, Shchechkin, and Tykhyy where a very important discovery of the conformal block interpretation of tau functions was made. For the history of the question, we refer the reader to the paper [ILP where the heuristic though truly pioneering results of [ILT13, [ILST] have been rigorously proven. Another conjectural pre-factor formula, this time concerning the third Painlevé equation (work [ILT14]), was proven in [IP]. Later on, the method of [ILP] and [IP] was succesfully applied to the first Painlevé equation in [LR].

The method of [ILP] and [IP] is inspired by the earlier works of B. Malgrange [Mal] and Bertola [Ber] and it is based on an extension of the Jimbo-Miwa-Ueno differential form $\omega_{\text {JMU }}$ to a 1-form, $\omega$ on the whole space $\mathscr{A} \simeq \widetilde{\mathscr{T}} \times \mathscr{M}$,

$$
\omega=\sum_{k=1}^{L} P_{k}(\vec{t}, M) d t_{k}+\sum_{j=1}^{d} Q_{j}(\vec{t}, M) d m_{j},
$$

such that

$$
\omega\left(\partial_{t_{k}}\right)=\omega_{\mathrm{JMU}}\left(\partial_{t_{k}}\right)
$$

and the exterior differential of $\omega$, i.e., the form,

$$
\Omega_{0}:=d \omega
$$

is a 2 -form on $\mathscr{M}$ only. Furthermore, it is independent of isomonodromic times $\mathscr{T}$. The construction of the form $\omega$ will be described in detail in the next section. 3

The time-independence of the 2-form $\Omega_{0}$ in conjunction with the Riemann-Hilbert computability of the asymptotics of $\Phi(z)$ determines what should be added to the form $\omega$ to make it closed, i.e. to transform it into the form $\hat{\omega}$ which satisfies the two crucial properties:

$$
d \hat{\omega} \equiv d_{\mathscr{T}} \hat{\omega}+d_{\mathcal{M}} \hat{\omega}=0, \quad \text { and } \quad \hat{\omega}\left(\partial_{t_{k}}\right)=\omega_{\mathrm{JMU}}\left(\partial_{t_{k}}\right) .
$$

Having the form $\hat{\omega}$, the tau function can be represented as

$$
\ln \tau=\int \hat{\omega} .
$$

Equation (8) allows one to use the asymptotic behavior of $\Phi(z)$ to evaluate the asymptotics of the associated tau function up to a numerical (i.e. independent of monodromy data) constant. The latter can be calculated by applying the final formulae to trivial solutions of deformation equations. This program has been first realized in [IP] for the sine-gordon reduction of the Painlevé III equation and later on in [ILP] for Painlevé VI and II equations and in $[\mathrm{LR}]$ for Painlevé I equation.

We want also mention the most recent work GL] where a general Fredholm determinant formula was found for the Painlevé VI tau function which allows to produce rigorously both the evaluation of the relevant asymptotic constants and the combinatorial series expansions of the tau function at the critical points.

\footnotetext{
${ }^{3}$ The exact relation of the form $\omega$ to the original Malgrange-Bertola 1-form is explained in detail in [ILP] -see Remark 4.4 there.
} 
In the course of the asymptotic analysis performed in $\overline{\text { IP }}$ and $[\mathrm{ILP}$, an interesting observation has been made with respect to the form $\omega$ in the Painlevé III and II cases. This observation is concerned with the Hamiltonian aspect of the theory of isomonodromic deformations which we have not yet discussed. As a matter of fact, the space $\mathscr{A}$ can be equipped with a symplectic structure - see $[\mathrm{H} 2]$, [Boal], [Kri], [B], so that the isomonodromic equation (6) induces $L$ commuting Hamiltonian flows on $\mathscr{A}$. A striking property of the tau function is that in many (though not al 4 known special cases its logarithm serves as the generating function of the Hamiltonians $H_{k}$ of these flows:

$$
\frac{\partial \ln \tau(\vec{t}, M)}{\partial t_{k}}=\left.\gamma H_{k}\right|_{A(z ; \vec{t}, M)} .
$$

Here $\gamma$ is numerical constant (in many cases, $\gamma=1$ ). This fact for the fourth, fifth and sixth Painlevé equations as well as for many higher rank isomonodromic systems was established in Boal2] where also a generalization of the JMU form allowing repeated eigenvalues was worked out. The Hamiltonian formalism for all six Painlevé equations was first suggested by K. Okamoto $\mathrm{O}$.

The above mentioned observation of [IP] and [ILP] is that in the Painlevé II and III cases the 2 -form $\Omega_{0}=d \omega$ is nothing else but, up to a numerical factor, the corresponding symplectic form. Hence, in these examples, the 1 -form $\omega$, up to a numerical factor and the addition of an explicit total differential, is an extension to the space $\widetilde{\mathscr{T}} \times \mathscr{M}$ of the differential of classical action; moreover, in IP and [LP these total differentials have been explicitly found. Similar relation to the classical action in the case of the Painlevé I tau-function has been obtained in $[\mathrm{LR}$, and in the case of the Schlesinger equations - the pure Fuchsian system (1), in [Mal2 5 .

The goal of this paper is to show that the relation between the tau function and the classical action established in [IP, [LP], and [LR for the special cases of Painlevé III, II, and I is true for all Painlevé equations. We shall also present some arguments allowing one to expect that this relation is, most likely, a general fact of the monodromy theory of linear systems.

The detailed construction of the form $\omega$ is given, following [ILP], in the next section. In this section we also provide the arguments in favor of the connection between the form $\omega$ and the classical action in the general case of linear system (1) and formulate two conjectures concerning with this connection. In Section 3 , these conjectures are justified for all six Painlevé equations and for an arbitrary Schlesinger system. In the cases of Painlevé I and Schlesinger equations we just reproduce the results of [LR and $[\mathrm{Mal}$, respectively.

\section{The extended Jimbo-Miwa-Ueno differential and the classical action functional.}

As in introduction, we shall, unless the otherwise is explicitly indicated, be treating all the objects which are defined on $\mathscr{A} \simeq \widetilde{\mathscr{T}} \times \mathscr{M}$ as functions of $(\vec{t}, M) \equiv(\vec{t}, \vec{m})$. In particular,

$$
\omega_{\mathrm{JMU}} \equiv \omega_{\mathrm{JMU}}(\vec{t}, M)=\left.\omega_{\mathrm{JMU}}\right|_{A(z ; \vec{t}, M)}
$$

and for any function $F$ on $\mathscr{A}$, the partial derivatives with respect to $t_{k}$ will mean the partial derivatives of $F$ as a function of $(\vec{t}, M)$, i.e.,

$$
\frac{\partial F}{\partial t_{k}} \equiv \frac{\partial}{\partial t_{k}} F(\vec{t}, M)=\frac{\partial}{\partial t_{k}}\left(\left.F\right|_{A(z ; \vec{t}, M)}\right)
$$

We will also use the notations

$$
d F \equiv d F(\vec{t}, M)=\sum_{k=1}^{L} \frac{\partial F}{\partial t_{k}} d t_{k}+\sum_{k=1}^{d} \frac{\partial F}{\partial m_{k}} d m_{k} \equiv d_{\mathscr{T}} F+d_{\mathscr{M}} F
$$

Our starting point is the following Lemma.

Lemma 1. ([JMU]) The 1-form (3) (considered as a 1-form on $\widetilde{\mathscr{T}} \times \mathscr{M}$ ) can be alternatively written as

$$
\omega_{\mathrm{JMU}}=\sum_{k=1}^{L} \sum_{v=1, \ldots, n, \infty} \operatorname{res}_{z=a_{v}} \operatorname{Tr}\left(A \frac{\partial G^{(v)}}{\partial t_{k}}\left(G^{(v)}\right)^{-1}\right) d t_{k} \equiv \sum_{v=1, \ldots, n, \infty} \operatorname{res}_{z=a_{v}} \operatorname{Tr}\left(A d_{\mathscr{T}} G^{(v)}\left(G^{(v)}\right)^{-1}\right) .
$$

\footnotetext{
${ }^{4}$ The statement actually depends on the specific choice of the symplectic structure.

${ }^{5}$ The authors are grateful to Marta Mazzocco for pointing out this result of B. Malgrange to us.
} 
We give the version [ILP] of the proof of this Lemma in Section 4.1 of the Appendix.

A direct corollary of Lemma円is the following integral formula for the tau function,

$$
\ln \tau \equiv \ln \tau\left(\vec{t}_{1}, \vec{t}_{2}, M\right)=\int_{\vec{t}_{1}}^{\vec{t}_{2}} \sum_{\nu, k} \operatorname{res}_{z=a_{v}} \operatorname{Tr}\left(A \frac{\partial G^{(v)}}{\partial t_{k}}\left(G^{(v)}\right)^{-1}\right) d t_{k} .
$$

Another consequence of the Lemma is the idea to take formula (10) as the motivation to introduce the following 1 -form (cf. [ILP], [IP)

$$
\begin{gathered}
\omega=\sum_{k=1}^{L} \sum_{v=1, \ldots, n, \infty} \operatorname{res}_{z=a_{v}} \operatorname{Tr}\left(A \frac{\partial G^{(v)}}{\partial t_{k}}\left(G^{(v)}\right)^{-1}\right) d t_{k}+\sum_{k=1}^{d} \sum_{v=1, \ldots, n, \infty} \operatorname{res}_{z=a_{v}} \operatorname{Tr}\left(A \frac{\partial G^{(v)}}{\partial m_{k}}\left(G^{(v)}\right)^{-1}\right) d m_{k} \\
\equiv \sum_{v=1, \ldots, n, \infty} \operatorname{res}_{z=a_{v}} \operatorname{Tr}\left(A d \mathscr{T} G^{(v)}\left(G^{(v)}\right)^{-1}\right)+\sum_{v=1, \ldots, n, \infty} \operatorname{res}_{z=a_{v}} \operatorname{Tr}\left(A d_{\mathcal{M}} G^{(v)}\left(G^{(v)}\right)^{-1}\right) \\
\equiv \sum_{v=1, \ldots, n, \infty} \operatorname{res}_{z=a_{v}} \operatorname{Tr}\left(A d G^{(v)}\left(G^{(v)}\right)^{-1}\right) .
\end{gathered}
$$

Now, the key observation.

Lemma 2 (ILP). The form $d \omega$ has no cross terms of the kind $d t_{k} \wedge d m_{j}, \quad k=1, \ldots, L, \quad j=1, \ldots, d$.

We present, following [ILP], the proof of this Lemma in section4.2 of the Appendix.

Lemma2plays a crucial role in the mentioned in the Introduction rigorous approach to the "constant problem". Indeed, a key issue in the determining of the monodromy dependence of the tau function is the possibility of the effective evaluation of the derivative of the integral (11) with respect to the monodromy parameters $m_{j}$. Lemma2implies that

$$
\frac{\partial}{\partial m_{j}} \sum_{v} \operatorname{res}_{z=a_{v}} \operatorname{Tr}\left(A \frac{\partial G^{(v)}}{\partial t_{k}}\left(G^{(v)}\right)^{-1}\right)=\frac{\partial}{\partial t_{k}} \sum_{v} \operatorname{res}_{z=a_{v}} \operatorname{Tr}\left(A \frac{\partial G^{(v)}}{\partial m_{j}}\left(G^{(v)}\right)^{-1}\right) .
$$

Therefore,

$$
\begin{gathered}
\frac{\partial \ln \tau}{\partial m_{j}}=\int_{\vec{t}_{1}}^{\vec{t}_{2}} \sum_{k=1}^{L} \frac{\partial}{\partial m_{j}} \sum_{v} \operatorname{res}_{z=a_{v}} \operatorname{Tr}\left(A \frac{\partial G^{(v)}}{\partial t_{k}}\left(G^{(v)}\right)^{-1}\right) d t_{k} \\
=\sum_{k=1}^{L} \int_{\vec{t}_{1}}^{\vec{t}_{2}} \frac{\partial}{\partial t_{k}} \sum_{v} \operatorname{res}_{z=a_{v}} \operatorname{Tr}\left(A \frac{\partial G^{(v)}}{\partial m_{j}}\left(G^{(v)}\right)^{-1}\right) d t_{k}=\left.\sum_{v} \operatorname{res}_{a_{v}} \operatorname{Tr}\left(A \frac{\partial G^{(v)}}{\partial m_{j}}\left(G^{(v)}\right)^{-1}\right)\right|_{\vec{t}_{1}} ^{\vec{t}_{2}}
\end{gathered}
$$

In other words, we conclude that in addition to the differential relation 44, i.e.,

$$
d_{\mathscr{T}} \ln \tau=\sum_{v=1, \ldots, n, \infty} \operatorname{res}_{z=a_{v}} \operatorname{Tr}\left(G^{(v)}(z)^{-1} A(z) d_{\mathscr{T}} G^{(v)}(z)\right),
$$

the tau function satisfies the differential relation,

$$
d_{\mathscr{M}} \ln \tau=\sum_{v=1, \ldots, n, \infty} \operatorname{res}_{z=a_{v}} \operatorname{Tr}\left(G^{(v)}(z)^{-1} A(z) d_{\mathscr{M}} G^{(v)}(z)\right) .
$$

These two differential identities allow to evaluate the asymptotic connection formulae up to the numerical constants and this is what is effectively done in [IP], [ILP].

The arguments which led to the representation (13) for the logarithmic derivative of the tau function with respect to $m_{j}$ are reminiscent to the variational equations for the classical action. Let us assume that we can 
identify the classical Darboux coordinates $6, p_{j}, q_{j}$ on the space $\mathscr{A}$ so that the isomonodromic deformation equations (6) can be written as the commuting system of Hamiltonian dynamical equations 7

$$
\frac{\partial q_{j}}{\partial t_{k}}=\frac{\partial H_{k}}{\partial p_{j}}, \quad \frac{\partial p_{j}}{\partial t_{k}}=-\frac{\partial H_{k}}{\partial q_{j}} .
$$

We remind that we are still identify $\mathscr{A} \simeq \widetilde{\mathscr{T}} \times \mathscr{M}$, so that we consider $p_{j}$ and $q_{j}$ as the functions on $\widetilde{\mathscr{T}} \times \mathscr{M}$,

$$
q_{j} \equiv q_{j}(\vec{t}, M), \quad p_{j} \equiv p_{j}(\vec{t}, M), \quad H_{k} \equiv H_{k}\left(q_{j}(\vec{t}, M), p_{j}(\vec{t}, M), \vec{t}\right) .
$$

The compatibility of the system [14] means (see, e.g., [B]) that all

$$
c_{k l}:=\left\{H_{k}, H_{l}\right\}+\frac{\partial H_{k}}{\partial t_{l}}-\frac{\partial H_{l}}{\partial t_{k}}
$$

are the Casimir function $\sqrt[8]{8}$ (maybe depending on the times $t_{k}$ ). We shall assume that

$$
c_{k l}=0 \quad \forall k, l .
$$

This assumption works for all example of the isomonodromic deformations equations that we know. The classical action differential can be defined as the differential form on $\widetilde{\mathscr{T}} \times \mathscr{M}$,

$$
\omega_{\text {cla }}=\sum p_{j} d q_{j}-\sum H_{k} d t_{k} \equiv \sum_{k}\left(\sum_{j} p_{j} \frac{\partial q_{j}}{\partial t_{k}}-H_{k}\right) d t_{k}+\sum_{k}\left(\sum_{j} p_{j} \frac{\partial q_{j}}{\partial m_{k}}\right) d m_{k}
$$

and, using assumption (15), it is easy to check that it is closed on the trajectories of the dynamical system [14, i.e.,

$$
d_{\mathscr{T}}\left(\left.\omega_{\text {cla }}\right|_{M \equiv \text { const }}\right)=0 .
$$

Note that in those cases when the logarithm of the tau function is the generating function for the Hamiltonians $H_{k}$, the Jimbo-Miwa-Ueno differential form is

$$
\omega_{\mathrm{JMU}}=\sum H_{k} d t_{k}
$$

so that the integral (11) is the truncated action integral,

$$
\ln \tau=\int_{\vec{t}_{1}}^{\vec{t}_{2}} \sum_{k} H_{k} d t_{k} .
$$

Suppose that instead of this integral we need to study the complete action, i.e. the integral,

$$
S \equiv S\left(\vec{t}_{1}, \vec{t}_{2}, M\right)=\int_{\vec{t}_{1}}^{\vec{t}_{2}} \omega_{\operatorname{cla}}(M) \equiv \int_{\vec{t}_{1}}^{\vec{t}_{2}} \sum_{k}\left(\sum_{j} p_{j} \frac{\partial q_{j}}{\partial t_{k}}-H_{k}\right) d t_{k} .
$$

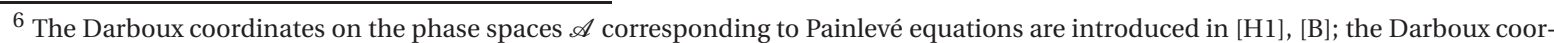
dinates for more general cases of the isomonodromic deformation equations are considered in $\mathrm{H2}$.

${ }^{7}$ In the special case of Painlevé and Schlesinger equations, which are our principal concern, their Hamiltonian representations [14] is described in all details in the main body of the paper. The interested reader can be referred to section 5 of Boal2 for the general definition of a time-dependent Hamiltonian system in the context of isomonodromy setting. We notice that this general definition is a delicate issue since the original parametrization of the extended phase space $\mathscr{A}$ mixes the time and dynamical parameters.

${ }^{8}$ Warning: here,

$$
\frac{\partial H_{k}}{\partial t_{l}}=\frac{\partial}{\partial t_{l}}\left(\left.H_{k}(\vec{p}, \vec{q}, \vec{t})\right|_{\vec{p}, \vec{q} \equiv \text { const }}\right) .
$$
}


Then, the usual variational calculus arguments show that, similar to [13), in any $m_{j}$-derivative of $S$ the integral terms would disappear. In fact, we have,

$$
\begin{gathered}
\frac{\partial S}{\partial m_{j_{0}}}=\int_{\vec{t}_{1}}^{\vec{t}_{2}} \sum_{k}\left(\sum_{j} \frac{\partial p_{j}}{\partial m_{j_{0}}} \frac{\partial q_{j}}{\partial t_{k}}+p_{j} \frac{\partial^{2} q_{j}}{\partial t_{k} \partial m_{j_{0}}}-\frac{\partial H_{k}}{\partial p_{j}} \frac{\partial p_{j}}{\partial m_{j_{0}}}-\frac{\partial H_{k}}{\partial q_{j}} \frac{\partial q_{j}}{\partial m_{j_{0}}}\right) d t_{k} \\
=\left.\sum_{j, k} p_{j} \frac{\partial q_{j}}{\partial m_{j_{0}}}\right|_{\vec{t}_{1}} ^{\vec{t}_{2}}+\int_{\vec{t}_{1}}^{\vec{t}_{2}} \sum_{k}\left(\sum_{j} \frac{\partial p_{j}}{\partial m_{j_{0}}} \frac{\partial q_{j}}{\partial t_{k}}-\frac{\partial q_{j}}{\partial m_{j_{0}}} \frac{\partial p_{j}}{\partial t_{k}}-\frac{\partial H_{k}}{\partial p_{j}} \frac{\partial p_{j}}{\partial m_{j_{0}}}-\frac{\partial H_{k}}{\partial q_{j}} \frac{\partial q_{j}}{\partial m_{j_{0}}}\right) d t_{k} \\
=\left.\sum_{j, k} p_{j} \frac{\partial q_{j}}{\partial m_{j_{0}}}\right|_{\vec{t}_{1}} ^{\vec{t}_{2}}
\end{gathered}
$$

and the integral term vanishes because of the equations of motion (14). Comparison (13) and (16) makes one to suspect some deep connection between the tau function and the classical action. And, indeed, taken the full exterior derivation of $\omega_{\mathrm{cla}} \equiv \omega_{\mathrm{cla}}(\vec{t}, M)$, one obtains,

$$
d \omega_{\mathrm{cla}}=\sum_{j} d_{\mathscr{M}} p_{j} \wedge d_{\mathscr{M}} q_{j} \equiv \Omega .
$$

The form $\Omega$ is the symplectic form associated with the dynamical system (14). Note, that both, the form $\Omega$ and the form $\Omega_{0}$ from $(7)$ are the closed 2- form on $\mathscr{M}$ and they do not depend on the times $\mathscr{T}$. This observation together with the similarities of the variational identities (13) and (16) allow us to formulate the following conjectures.

Conjecture 1. Suppose that the parameter space $\mathscr{A}$ is equipped with the symplectic structure. Let $\Omega$ be a corresponding symplectic form and $\Omega_{0}$ be the two-form defined in $\mathbf{7 7}$. Then, there exists a numerical constant $\gamma$ such that,

$$
\Omega_{0}=\gamma \Omega
$$

If this conjecture is true then the two 1-forms, $\omega$ and $\omega_{\text {cla }}$, coincide up to the total differential. Hence our next conjecture

Conjecture 2. There exists a rational function $G(\vec{p}, \vec{q}, \vec{t})$ of $\vec{p}, \vec{q}, \vec{t}$ such that,

$$
\omega=\gamma \omega_{\mathrm{cla}}+d G(\vec{p}, \vec{q}, \vec{t}) .
$$

\section{Moreover, the function $G(\vec{p}, \vec{q}, \vec{t})$ is explicitly computable.}

As it has already been indicated in the Introduction, the statement of Conjecture 2 has been proven to be true in the case of the Schlesinger equations [Mal2], in the case of the sine-gordon reduction of Painleve III equation [IP], in the case of the (homogenous) Painlevé II equation [ILP], and in the case of the Painlevé I equation $[\overline{\mathrm{LR}}$. In the next section of this paper we demonstrate the validity of the both conjectures for the rest of the Painlevé equations, and we also present, for completeness, the result of Mal2.

Remark 3. Restricting [17 to the isomonodromic family $M \equiv$ const, one arrives to the identity

$$
\begin{gathered}
\sum_{k} \frac{\partial \ln \tau}{\partial t_{k}} d t_{k}=\sum_{k}\left(\sum_{j} p_{j}(\vec{t}, M) \frac{\partial q_{j}(\vec{t}, M)}{\partial t_{k}}-H_{k}(\vec{p}(\vec{t}, M), \vec{q}(\vec{t}, M), \vec{t})\right) d t_{k} \\
+\sum_{k} \frac{\partial}{\partial t_{k}} G(\vec{p}(\vec{t}, M), \vec{q}(\vec{t}, M), \vec{t}) d t_{k} .
\end{gathered}
$$

and hence,

$$
\ln \tau\left(\vec{t}_{1}, \vec{t}_{2}, M\right)=S\left(\vec{t}_{1}, \vec{t}_{2}, M\right)+\left.G(\vec{p}(\vec{t}, M), \vec{q}(\vec{t}, M), \vec{t})\right|_{\vec{t}_{1}} ^{\vec{t}_{2}}
$$


This, in turn, would produce, taking into account (16), the following, alternative to (13), formula for the $m_{j}$ derivative of $\ln \tau$,

$$
\frac{\partial \ln \tau}{\partial m_{j_{0}}}=\left.\sum_{j, k} p_{j} \frac{\partial q_{j}}{\partial m_{j_{0}}}\right|_{\vec{t}_{1}} ^{\vec{t}_{2}}+\left.\frac{\partial G}{\partial m_{j_{0}}}\right|_{\vec{t}_{1}} ^{\vec{t}_{2}} .
$$

This version of the variational logarithmic derivatives of the tau functions turns out even more efficient then (13) in the concrete examples related to the "constant problem". Indeed, the particular cases of (20) have been used in [BIP] in evaluation of the constant terms in the asymptotics of the several basic distribution functions of random matrix theory expressible in terms of the Painlevé transcendents.

Remark 4. In the pioneering papers [ILST] and [ILT13], the evaluation of the constant pre-factors has been partially based on the conjectural interpretations of these constant factors as the generating functions of the canonical transformations between the Darboux asymptotic coordinates associated with the different critical points. This generating function interpretation of the constant pre-factors, which in the case of Painlevé II, III and I has been proven in [IP, ILP] and [LR], can be considered as a direct corollary of [19].

\section{Painlevé equations}

In this section we present the exact realization of the relations $\sqrt{17}-\sqrt{18}$ between the tau-function and the classical action for all six Painlevé equations and also for the case of the general Schlesinger equation. We shall start with the second Painlevé equation which we take as a case study and illustrate in detail the general constructions of Section 2 In particular, we will describe exactly the both spaces, $\mathscr{A}$ and $\mathscr{M}$ corresponding to the Painlevé II equation. The other Painlevé equations will be treated with less details. We won't describe the space $\mathscr{M}$ for other Painlevé equations explicitly, and instead we will refer the reader either to [JM] or to Chapter 5 of FIKN.

\subsection{Painlevé II}

According to [JM], the second Painlevé equation describes the isomonodromic deformations of the $2 \times 2$ linear system having only one irregular singular point at $z=\infty$ of the Poincaré rank 3 ,

$$
\frac{d \Phi}{d z}=A(z) \Phi, \quad A(z)=A_{2} z^{2}+A_{1} z+A_{0} .
$$

Following again $[\mathrm{JM}]$, we normalize the system by the conditions,

$$
\operatorname{Tr} A(z) \equiv 0, \quad A_{2}=\left(\begin{array}{cc}
1 & 0 \\
0 & -1
\end{array}\right), \quad A_{1,11}=A_{1,22}=0,
$$

so that the matrix coefficients $A_{1}$ and $A_{0}$ can be written in the form,

$$
A_{1}=\left(\begin{array}{cc}
0 & k \\
-\frac{2 p}{k} & 0
\end{array}\right), \quad A_{0}=\left(\begin{array}{cc}
p+\frac{t}{2} & -k q \\
-\frac{2}{k}(\theta+p q) & -p-\frac{t}{2}
\end{array}\right),
$$

and the complex parameters $p, q, k, \theta$, and $t$ can be taken as the original coordinates on the corresponding space $\mathscr{A}$,

$$
\mathscr{A}=\{(p, q, k, \theta, t)\} .
$$

The formal solution of system (21) at its only (irregular) singular point, $z=\infty$, has the structure (cf. (2)),

$$
\begin{gathered}
\Phi_{\text {form }}(z) \equiv G(z) e^{\Theta(z)} \\
=\left(I+\frac{g_{1}}{z}+\frac{g_{2}}{z^{2}}+\frac{g_{3}}{z^{3}}+\ldots\right) e^{\Theta(z)}, \quad \Theta(z)=\sigma_{3}\left(\frac{z^{3}}{3}+\frac{t z}{2}-\theta \ln z\right),
\end{gathered}
$$

with the first three matrix coefficients $g_{k}, k=1,2,3$ given as functions on the space $\mathscr{A}$ (22) by the explicit formulae,

$$
g_{1}=\left(\begin{array}{cc}
-H & -\frac{k}{2} \\
-\frac{p}{k} & H
\end{array}\right)
$$




$$
\begin{gathered}
g_{2}=\left(\begin{array}{cc}
\frac{H^{2}}{2}+\frac{p}{4}-\frac{t \theta}{4} & -\frac{k H}{2}+\frac{k q}{2} \\
\frac{p H}{k}-\frac{p q}{k}-\frac{\theta}{k} & \frac{H^{2}}{2}+\frac{p}{4}+\frac{t \theta}{4}
\end{array}\right), \\
g_{3}=\left(\begin{array}{cc}
-\frac{H^{3}}{6}-\frac{H p}{4}+\frac{H t}{6}+\frac{H t \theta}{4}+\frac{p q}{6}+\frac{\theta^{2}}{6}+\frac{\theta}{3} & -\frac{k H^{2}}{4}+\frac{k q H}{2}+\frac{k p}{8}+\frac{k t}{4}-\frac{k t \theta}{8} \\
-\frac{p H^{2}}{2 k}+\frac{H p q}{k}+\frac{H \theta}{k}+\frac{p^{2}}{4 k}+\frac{p t \theta}{4 k}+\frac{p t}{2 k} & \frac{H^{3}}{6}+\frac{H p}{4}-\frac{H t}{6}+\frac{H t \theta}{4}-\frac{p q}{6}-\frac{\theta^{2}}{6}+\frac{\theta}{6}
\end{array}\right),
\end{gathered}
$$

where

$$
H=\frac{p^{2}}{2}+p q^{2}+\frac{p t}{2}+q \theta
$$

The system [21] has seven canonical solutions, characterized by the asymptotic condition,

$$
\Phi_{j}(z) \simeq \Phi_{\text {form }}(z), \quad z \rightarrow \infty, \quad \frac{(2 j-5) \pi}{6}<\arg z<\frac{(2 j-1) \pi}{6}, \quad \Phi_{7}(z)=\Phi_{1}(z) e^{-2 \pi i \theta \sigma_{3}} .
$$

and hence it has six Stokes matrices, $S_{j}=\Phi_{j}^{-1}(z) \Phi_{j+1}(z)$, which have the following triangular structure ( for more detail see [JM] or Chapter 5 of [FIKN]),

$$
S_{2 k+1}=\left(\begin{array}{cc}
1 & 0 \\
s_{2 k+1} & 1
\end{array}\right), \quad S_{2 k}=\left(\begin{array}{cc}
1 & s_{2 k} \\
0 & 1
\end{array}\right),
$$

and satisfy one cyclic relation,

$$
S_{1} S_{2} \ldots S_{6}=e^{-2 \pi i \theta \sigma_{3}} .
$$

Also, as it follows from (23), the parameter $\theta$ determines the formal monodromy exponent, $\Theta_{0}=\theta \sigma_{3}$. This means, that the space $\mathscr{M}$ in the case of system (21) can be identified with the algebraic variety of dimension 4 ,

$$
\mathscr{M}=\left\{\vec{m}=\left(s_{1}, s_{2}, \ldots, s_{6}, \theta\right): 1+s_{1} s_{2}=\left(1+s_{4} s_{5}\right) e^{2 \pi i \theta}, 1+s_{2} s_{3}=\left(1+s_{5} s_{6}\right) e^{-2 \pi i \theta}, s_{1}+s_{3}+s_{1} s_{2} s_{3}=-s_{5} e^{2 \pi i \theta}\right\} .
$$

Equation [23], also tells us that the parameter $t$ is the only isomonodromic time, so that, in the case of system (21) we have,

$$
\mathscr{T}=\{t\} .
$$

The isomonodromic deformations of system [21, i.e., the conditions,

$$
p=p(t, M), q=q(t, M), k=k(t, M), \quad M \equiv \text { const },
$$

yield the second linear matrix differential equation, this time with respect to $t$, for the function $\Phi(z) \equiv$ $\Phi(z ; t, M)$,

$$
\frac{d \Phi}{d t}=B(z) \Phi, \quad B(z)=B_{1} z+B_{0},
$$

where

$$
B_{1}=\frac{1}{2}\left(\begin{array}{cc}
1 & 0 \\
0 & -1
\end{array}\right), \quad B_{0}=\frac{1}{2}\left(\begin{array}{cc}
0 & k \\
-\frac{2 p}{k} & 0
\end{array}\right) .
$$

Equations (21) and (28) form a Lax pair (cf. (5)),

$$
\left\{\begin{array}{l}
\frac{d \Phi}{d z}=A(z) \Phi \\
\frac{d \Phi}{d t}=B(z) \Phi
\end{array}\right.
$$

whose compatibility condition (6), in terms of the functional parameters $p, q, k$ and $\theta$ reads

$$
\begin{aligned}
& \frac{d q}{d t}=p+q^{2}+\frac{t}{2}, \\
& \frac{d p}{d t}=-2 p q-\theta, \\
& \frac{d k}{d t}=-k q, \\
& \frac{d \theta}{d t}=0 .
\end{aligned}
$$


The last equation of this system is just the statement that $\theta$, as the part of the monodromy data, is constant. The third equation gives $\ln k(t)$ as the antiderivative of $-q(t)$. The first two first order differential equations are equivalent to one second order differential equation, indeed, the Painlevé II equation,

$$
q_{t t}=t q+2 q^{3}+\alpha, \quad \alpha=\frac{1}{2}-\theta .
$$

Assuming that

$$
\theta \equiv \text { const }
$$

one can easily see that the function [27) is the Hamiltonian of the second Painleve equation $[30$ ) with respect to the symplectic form,

$$
\Omega=d p \wedge d q,
$$

Indeed, the first and the second equations in (29) are just

$$
\frac{d q}{d t}=\frac{\partial H}{\partial p}, \quad \text { and } \quad \frac{d p}{d t}=-\frac{\partial H}{\partial q},
$$

respectively.

Let us now discuss the forms $\omega_{\mathrm{JMU}}$ and $\omega$ corresponding to (21). The linear system (21) has only $\infty$ as its singular point. Therefore, the general definition [3] of the form $\omega_{\mathrm{JMU}}$ transforms to the equation,

$$
\omega_{\mathrm{JMU}}=-\operatorname{res}_{z=\infty} \operatorname{Tr}\left(G^{-1}(z) \frac{d G(z)}{d z} \frac{d \Theta(z)}{d t}\right) d t .
$$

Plugging 23 at the right hand side we arrive at the formulae,

$$
\omega_{\mathrm{JMU}}=-\operatorname{Tr}\left(\frac{1}{2} g_{1} \sigma_{3}\right),
$$

or, taking into account 24) (cf. (9),

$$
\omega_{\mathrm{JMU}} \equiv \frac{d \ln \tau}{d t} d t=H d t,
$$

Similarly, the general definition (12) of the form $\omega$ transforms to the equation,

$$
\omega=\operatorname{res}_{z=\infty} \operatorname{Tr}\left(A(z) d G(z) G(z)^{-1}\right) .
$$

Plugging (23) into [32] we arrive at the formula,

$$
\omega=\operatorname{Tr}\left(A_{2} d g_{3}-A_{2} d g_{2} g_{1}-A_{2} d g_{1} g_{2}+A_{2} d g_{1} g_{1}^{2}+A_{1} d g_{2}-A_{1} d g_{1} g_{1}+A_{0} d g_{1}\right) .
$$

Now, it is more involved to plug (24) - (26) into the right hand side of the last equation. However, after performing some algebra, the final expression comes out rather simple,

$$
\omega=-\frac{1}{3} q d p+\frac{2}{3} p d q-\theta \frac{d k}{k}+\frac{2}{3} t d H-\frac{1}{3} H d t-\frac{2 \theta-1}{3} d \theta,
$$

and can be in turn easily transformed to the equation,

$$
\omega=p d q-H d t+d\left(\frac{2}{3} H t-\frac{1}{3} q p-\theta \ln k-\frac{\theta^{2}}{3}+\frac{\theta}{3}\right)+\ln k d \theta .
$$

If we again assume that

$$
\theta \equiv \text { const }
$$

relation (33) reduces to

$$
\omega=p d q-H d t+d\left(\frac{2}{3} H t-\frac{1}{3} q p-\theta \ln k\right) .
$$


Equation 34 proves Conjecture 2 in the case of the $2 \times 2$ system (21) with the additional constraint, $\theta \equiv$ constant. Indeed, this is exactly the formula [17) with the specification 9

$$
G(p, q, t)=\frac{2}{3} H t-\frac{1}{3} q p-\theta \ln k .
$$

The corresponding equation 18 is

$$
\begin{gathered}
\frac{d \ln \tau}{d t}=p \frac{d q}{d t}-H+\frac{d}{d t}\left(\frac{2}{3} H t-\frac{1}{3} q p-\theta \ln k\right) \\
=p \frac{d q}{d t}-H+\frac{d}{d t}\left(\frac{2}{3} H t-\frac{1}{3} q p\right)+\theta q .
\end{gathered}
$$

We also note that Conjecture 1 follows directly from 34.

Remark 5. Together with [31, equation (35) is just an identity which can be proven directly by substituting (31) into the left hand side of [35]. However, it would be quite difficult to guess, without having the concept of the form $\omega$, the existence of such connection between the truncated and the full action differentials. We believe that the fact that in the case of the Painlevé dynamical systems the truncated action differs from the full action by a total differential is a manifestation of their Lax-pair integrability.

Remark 6. Let us denote

$$
p_{1}=p, \quad q_{1}=q, \quad p_{2}=\ln k, \quad q_{2}=\theta,
$$

then the whole system (29) becomes Hamiltonian with the same Hamiltonian 27,

$$
H=\frac{p_{1}^{2}}{2}+p_{1} q_{1}^{2}+\frac{p_{1} t}{2}+q_{1} q_{2}
$$

and with respect to the symplectic form,

$$
\Omega=d p_{1} \wedge d q_{1}+d p_{2} \wedge d q_{2}
$$

Moreover, 34) can be written as

$$
\omega=\sum_{k=1}^{2} p_{k} d q_{k}-H d t+d\left(\frac{2}{3} H t-\frac{1}{3} q_{1} p_{1}-q_{2} p_{2}-\frac{q_{2}^{2}}{3}+\frac{q_{2}}{3}\right) .
$$

That is, if we associate with the linear system $[21$ not just the second Painlevé equation 30 but the full system (29) of the isomonodromic deformation equations of [21), then the G-function in the relation (17) will be totally local and, in fact, polynomial in the Darboux coordinates,

$$
G\left(p_{1}, p_{2}, q_{1}, q_{2}, t\right)=\frac{2}{3} H t-\frac{1}{3} q_{1} p_{1}-q_{2} p_{2}-\frac{q_{2}^{2}}{3}+\frac{q_{2}}{3} .
$$

It is also worth noticing that since $H$ does not contain $p_{2}$, the variable $q_{2}$ is an action variable, i.e., it is constant, as it should be.

Remark 7. The Lax pair [21], 28] is not the only Lax pair for the Painlevé II equation. If we use another Lax pair, for instance the Lax pair of Flaschka and Newell $[\overline{F N}]$, we would get the another tau function, another Hamiltonian and another form $\omega$. However, the Conjectures 1 and 2 would still be true - see [ILP] and [BIP]. It is an interesting issue how much the Hamiltonian aspects we are promoting depend on the concrete Lax pair realization of the Painlevé equations.

\footnotetext{
${ }^{9}$ From the point of view of the asymptotic analysis of the tau functions outlined in the Introduction, the appearance of the non-local term $\ln k=\int q d t$ is not an obstacle since the functional parameter $k$ equals $-2 g_{1,12}$ (see 24]) where $g_{1}$ is the first coefficient of the series 23. This means that, similar to the functions $p$ and $q$, it can be recovered from the underlining Riemann-Hilbert problem, see also BBDI.
} 


\subsection{Painlevé I}

The results of this subsections belong to O. Lisovyy and J. Roussillon, and we follow here their paper [LR]. The linear system associated with the first Painlevé equation is the $2 \times 2$ matrix ODE with one irregular singular point of Poincaré rank 5 at $z=\infty$ and with one Fuchsian singular point at $z=0$,

$$
\frac{d \Phi}{d z}=A(z) \Phi, \quad A(z)=A_{4} z^{4}+A_{2} z^{2}+A_{1} z+A_{0}+\frac{A_{-1}}{z} .
$$

The matrix coefficients are,

$$
A_{4}=\left(\begin{array}{cc}
4 & 0 \\
0 & -4
\end{array}\right), \quad A_{2}=\left(\begin{array}{cc}
0 & -4 q \\
4 q & 0
\end{array}\right), \quad A_{1}=\left(\begin{array}{cc}
0 & -2 p \\
-2 p & 0
\end{array}\right), \quad A_{0}=\left(\begin{array}{c}
2 q^{2}+t-2 q^{2}-t \\
2 q^{2}+t-2 q^{2}-t
\end{array}\right), \quad A_{-1}=-\frac{1}{2}\left(\begin{array}{ll}
0 & 1 \\
1 & 0
\end{array}\right)
$$

and so that the space $\mathscr{A}$ is parametrized by $p, q$, and $t$,

$$
\mathscr{A}=\{(p, q, t)\} .
$$

The formal solution at $z=\infty$ is given by the series,

$$
\Phi_{\text {form }}(z)=\left(I+\frac{g_{1}}{z}+\frac{g_{2}}{z^{2}}+\frac{g_{3}}{z^{3}}+\frac{g_{4}}{z^{4}}+\frac{g_{5}}{z^{5}}+O\left(\frac{1}{z^{6}}\right)\right) e^{\Theta(z)}, \quad \Theta(z)=\sigma_{3}\left(\frac{4 z^{5}}{5}+t z\right)
$$

with the explicit formulae for the first five coefficient matrices $g_{k}$ given by the equations,

$$
\begin{gathered}
g_{1}=\left(\begin{array}{cc}
-H & 0 \\
0 & H
\end{array}\right), \quad g_{2}=\left(\begin{array}{cc}
\frac{H^{2}}{2} & \frac{q}{2} \\
\frac{q}{2} & \frac{H^{2}}{2}
\end{array}\right), \quad g_{3}=\left(\begin{array}{cc}
-\frac{H^{3}}{6}-\frac{2 p-t^{2}}{24} & \frac{q H}{2}+\frac{p}{4} \\
-\frac{q H}{2}-\frac{p}{4} & \frac{H^{3}}{6}+\frac{2 p-t^{2}}{24}
\end{array}\right), \\
g_{4}=\left(\begin{array}{cc}
\frac{H^{4}}{24}+\frac{2 p-t^{2}}{24} H+\frac{q^{2}}{8} & \frac{q H^{2}}{4}+\frac{p H}{4}+\frac{2 q^{2}+t}{8} \\
\frac{q H^{2}}{4}+\frac{p H}{4}+\frac{2 q^{2}+t}{8} & \frac{H^{4}}{24}+\frac{2 p-t^{2}}{24} H+\frac{q^{2}}{8}
\end{array}\right), \\
g_{5}=\left(\begin{array}{cc}
-\frac{H^{5}}{120}-\frac{2 p-t^{2}}{48} H^{2}-\frac{5 q^{2}-2 t}{40} H-\frac{4 p q+1}{160} & \frac{q H^{3}}{12}+\frac{p H^{2}}{8}+\frac{2 q^{2}+t}{8} H+\frac{2 p-t^{2}}{48} q+\frac{1}{16} \\
-\frac{q H^{3}}{12}-\frac{p H^{2}}{8}-\frac{2 q^{2}+t}{8} H-\frac{2 p-t^{2}}{48} q-\frac{1}{16} & \frac{H^{5}}{120}+\frac{2 p-t^{2}}{48} H^{2}+\frac{5 q^{2}-2 t}{40} H+\frac{4 p q+1}{160}
\end{array}\right) .
\end{gathered}
$$

where

$$
H=\frac{p^{2}}{2}-2 q^{3}-t q
$$

The Fuchsian point $z=0$ is a resonant point and hence the generic theory outlined in the Introduction is not applicable. In fact, the behavior of the solution $\Phi(z)$ at $z=0$ is given by the formula [LR],

$$
\Phi(z)=\left(\begin{array}{cc}
1 & \frac{1}{2} \\
1 & -\frac{1}{2}
\end{array}\right) z^{-\frac{1}{2} \sigma_{3}} \hat{\Phi}(z)
$$

where $\hat{\Phi}(z)$ is holomorphic and invertible at $z=0$.

The set of monodromy data $\mathscr{M}$ of system 36 consists of ten Stokes matrices associated with the irregular singularity at $z=\infty$ out of which, due to the symmetry $z \rightarrow-z$ of the system, only two are in fact free, i.e. $\operatorname{dim} \mathscr{M}=2$ (see $[\overline{\mathrm{LR}}]$ for details). The structure of the essential singularity of $\Phi(z)$ at infinity described in (37) indicates that the parameter $t$ is the only isomonodromic time. The corresponding Lax pair is formed by equation (36) and the following additional matrix equation,

$$
\begin{array}{cc}
\frac{d \Phi}{d t}=B(z) \Phi, & B(z)=B_{1} z+\frac{B_{-1}}{z}, \\
B_{1}=\left(\begin{array}{cc}
1 & 0 \\
0 & -1
\end{array}\right), & B_{-1}=\left(\begin{array}{c}
q-q \\
q-q
\end{array}\right) .
\end{array}
$$


The compatibility condition of (36) and (42) yields the system of ODEs on $q \equiv q(t)$ and $p \equiv p(t)$,

$$
\begin{aligned}
& \frac{d q}{d t}=p, \\
& \frac{d p}{d t}=6 q^{2}+t .
\end{aligned}
$$

which is equivalent to the Painlevé I equation

$$
q_{t t}=6 q^{2}+t
$$

The system (29) is a Hamiltonian system with the Hamiltonian 41].

We are passing now to the forms $\omega_{\mathrm{JMU}}$ and $\omega$ corresponding to the Painlevé I system [29). Because $z=0$ is the resonant Fuchsian point we strictly speaking can not use the definitions (3) and (12) for the forms $\omega_{\text {JMU }}$ and $\omega$. However, following [LR], we take (3) and (12), where the undefined contribution of the resonant point $z=0$ simply ignored, as the definitions of these forms in the case of the Painlevé I equations, i.e., as in the case of the second Painlevé equation, we put

$$
\omega_{\mathrm{JMU}}=-\operatorname{res}_{z=\infty} \operatorname{Tr}\left(G^{-1}(z) \frac{d G(z)}{d z} \frac{d \Theta(z)}{d t}\right) d t,
$$

and

$$
\omega=\operatorname{res}_{z=\infty} \operatorname{Tr}\left(A(z) d G(z) G(z)^{-1}\right),
$$

where $G(z)$ and $\Theta(z)$ are the series and the exponent from [37. As it is shown in [LR] such approach preserves the validity of Lemmas 1 1and 2 Just as in the case of Painevé II, we obtain from 44] at once that

$$
\omega_{\mathrm{JMU}} \equiv \frac{d \ln \tau}{d t} d t=2 H d t .
$$

The form $\omega$ needs more work.

Introduce the matrix coefficients $h_{j}$ of the series inverse to the series [37,

$$
\left(I+\frac{h_{1}}{z}+\frac{h_{2}}{z^{2}}+\frac{h_{3}}{z^{3}}+\frac{h_{4}}{z^{4}}+O\left(\frac{1}{z^{5}}\right)\right):=\left(I+\frac{g_{1}}{z}+\frac{g_{2}}{z^{2}}+\frac{g_{3}}{z^{3}}+\frac{g_{4}}{z^{4}}+\frac{g_{5}}{z^{5}}+O\left(\frac{1}{z^{6}}\right)\right)^{-1} .
$$

We have for the first four coefficients the relations,

$$
\begin{aligned}
& h_{1}=-g_{1}, \quad h_{2}=-g_{2}+g_{1}^{2}, \quad h_{3}=-g_{3}+g_{2} g_{1}+g_{1} g_{2}-g_{1}^{3}, \\
& h_{4}=-g_{4}+g_{3} g_{1}+g_{1} g_{3}+g_{2}^{2}-g_{2} g_{1}^{2}-g_{1} g_{2} g_{1}-g_{1}^{2} g_{2}+g_{1}^{4} .
\end{aligned}
$$

Plugging (46) and (37) into 45) we arrive at the formula,

$$
\omega=\operatorname{Tr}\left(-A_{4}\left(h_{4} d g_{1}+h_{3} d g_{2}+h_{2} d g_{3}+h_{1} d g_{4}+d g_{5}\right)-A_{2}\left(h_{2} d g_{1}+h_{1} d g_{2}+d g_{3}\right)-A_{1}\left(h_{1} d g_{1}+d g_{2}\right)-A_{0} d g_{1}\right) .
$$

Using (38)-40) we get after (quite a lot of) simplifications

$$
\omega=\frac{6}{5} p d q-\frac{4}{5} q d p-\frac{2}{5} H d t+\frac{8}{5} t d H,
$$

and properly combining the terms,

$$
\omega=2\left[p d q-H d t+d\left(\frac{4 H t}{5}-\frac{2 p q}{5}\right)\right] .
$$

Equation (47) proves Conjectures 1 and 2 , with $\gamma=2$, in the case of the $2 \times 2$ system (36) and gives the explicit formula for $G(p, q, t)$,

The corresponding equation $[18)$ is

$$
G(p, q, t)=\frac{2}{5}(4 H t-2 p q) .
$$

$$
\frac{d \ln \tau}{d t}=2\left(p \frac{d q}{d t}-H\right)+\frac{2}{5} \frac{d}{d t}(4 H t-2 p q),
$$

and, of course, can be easily checked directly. 


\subsection{Painlevé III}

The linear system associated with the third Painlevé equation we take again from $[\overline{\mathrm{JM}}]$. This is the system,

$$
\frac{d \Phi}{d z}=A(z) \Phi, \quad A(z)=\frac{A_{-2}}{z^{2}}+\frac{A_{-1}}{z}+A_{0}
$$

with

$$
A_{0}=\frac{1}{2}\left(\begin{array}{cc}
t & 0 \\
0 & -t
\end{array}\right), \quad A_{-1}=\left(\begin{array}{cc}
-\theta_{\infty} & -q k t \\
\frac{p q(t-p)}{k t}+\frac{\theta_{0}+\theta_{\infty}}{k}-\frac{2 \theta_{\infty} p}{k t} & \theta_{\infty}
\end{array}\right), \quad A_{-2}=\left(\begin{array}{cc}
p-\frac{t}{2} & -k t \\
\frac{p(p-t)}{k t}-p+\frac{t}{2}
\end{array}\right) .
$$

The system has two irregular singular points at $z=\infty$ and $z=0$, both of the Poincaré rank 1 . The corresponding formal solutions are:

$$
\Phi_{\text {form }}^{(\infty)}(z)=\left(I+\frac{g_{\infty, 1}}{z}+O\left(\frac{1}{z^{2}}\right)\right) e^{\Theta_{\infty}(z)}, \quad \Theta_{\infty}(z)=\sigma_{3}\left(\frac{t z}{2}-\theta_{\infty} \ln z\right)
$$

with

$$
g_{\infty, 1}=\left(\begin{array}{cc}
-\frac{H}{2}-\frac{p q}{2 t}+\frac{\theta_{\infty}^{2}-\theta_{0}^{2}}{2 t}+\frac{t}{2} & k q \\
\frac{p q(t-p)}{k t^{2}}+\frac{\theta_{0}+\theta_{\infty}}{k t}-\frac{2 \theta_{\infty} p}{k t^{2}} & \frac{H}{2}+\frac{p q}{2 t}-\frac{\theta_{\infty}^{2}-\theta_{0}^{2}}{2 t}-\frac{t}{2}
\end{array}\right),
$$

at $z=\infty$, and

$$
\Phi_{\text {form }}^{(0)}(z)=G_{0}\left(I+g_{0,1} z+O\left(z^{2}\right)\right) e^{\Theta_{0}(z)}, \quad \Theta_{0}(z)=\sigma_{3}\left(\frac{t}{2 z}+\theta_{0} \ln z\right)
$$

with

$$
g_{0,1}=\left(\begin{array}{cc}
-\frac{H}{2}-\frac{p q}{2 t}-\frac{\theta_{\infty}^{2}-\theta_{0}^{2}}{2 t}+\frac{t}{2} & \frac{k q a}{t}(p-t)+\frac{k a}{t}\left(\theta_{\infty}-\theta_{0}\right) \\
-\frac{1}{k t a}\left(p q+\theta_{0}+\theta_{\infty}\right) & \frac{H}{2}+\frac{p q}{2 t}+\frac{\theta_{\infty}^{2}-\theta_{0}^{2}}{2 t}-\frac{t}{2}
\end{array}\right)
$$

at $z=0$. In 50 and $[52$,

$$
H=\frac{1}{t}\left(2 p^{2} q^{2}+p\left(2 t-2 t q^{2}+\left(4 \theta_{\infty}-1\right) q\right)-2 t q\left(\theta_{0}+\theta_{\infty}\right)+\theta_{\infty}^{2}-\theta_{0}^{2}\right),
$$

and $G_{0}$ diagonalizes matrix $A_{-2}$,

$$
G_{0}^{-1} A_{-2} G_{0}=-\frac{t \sigma_{3}}{2}
$$

and it is chosen in the form

$$
G_{0}=\frac{1}{\sqrt{k}}\left(\begin{array}{cc}
k & -k \\
\frac{p}{t} & \frac{t-p}{t}
\end{array}\right) a^{-\frac{\sigma_{3}}{2}},
$$

with $a$ being an extra gauge parameter, so that the full space $\mathscr{A}$ is seven dimensional,

$$
\mathscr{A}=\left\{p, q, k, a, t, \theta_{0}, \theta_{\infty}\right\}
$$

From the series [49] and [51] it follows that $\theta_{\infty}$ and $\theta_{0}$ are the formal monodromy exponents and the parameter $t$ is the isomonodromic time. The isomonodromicity with respect to $t$ yields the second differential equation for $\Phi(z) \equiv \Phi(z, t)$,

$$
\frac{d \Phi}{d t}=B(z) \Phi, \quad B(z)=B_{1} z+B_{0}+\frac{B_{-1}}{z},
$$

where,

$$
B_{1}=\frac{1}{2}\left(\begin{array}{cc}
1 & 0 \\
0 & -1
\end{array}\right), \quad B_{0}=\frac{1}{t}\left(\begin{array}{cc}
0 & -q k t \\
\frac{p q(t-p)}{k t}+\frac{\theta_{0}+\theta_{\infty}}{k}-\frac{2 \theta_{\infty} p}{k t} & 0
\end{array}\right), \quad B_{-1}=\left(\begin{array}{cc}
\frac{t-2 p}{2 t} & k \\
\frac{p(t-p)}{k t^{2}} & \frac{2 p-t}{2 t}
\end{array}\right) .
$$


The compatibility condition of the matrix equations 48 and 56 implies the following dynamical system on [55),

$$
\begin{aligned}
& \frac{d q}{d t}=\frac{4 p q^{2}}{t}-2 q^{2}+\frac{q\left(4 \theta_{\infty}-1\right)}{t}+2, \\
& \frac{d p}{d t}=-\frac{4 p^{2} q}{t}+\frac{p\left(4 t q-4 \theta_{\infty}+1\right)}{t}+2 \theta_{0}+2 \theta_{\infty}, \\
& \frac{d k}{d t}=-\frac{4 p q k}{t}+2 q k-\frac{2 \theta_{\infty} k}{t}, \quad \frac{d a}{d t}=\frac{a}{t}\left(2 q t+2 \theta_{0}\right), \\
& \frac{d \theta_{\infty}}{d t}=0, \quad \frac{d \theta_{0}}{d t}=0 .
\end{aligned}
$$

It should be also mentioned, that the fourth equation, i.e. the equation for the function $a(t)$, follows from plugging [51] into equation [56 - the second equation of the Lax pair, and equating the terms of zero order with respect to $z$.

The last two equations of system (57) just state that $\theta_{\infty}$ and $\theta_{0}$ as the part of the monodromy data, are constant. The third and the fourth equations give $\ln k(t)$ and $\ln a(t)$ as the antiderivatives of the simple combinations of $p$ and $q$. The first two equations are equivalent to the third Painlevé equation,

$$
q_{t t}=\frac{\left(q_{t}\right)^{2}}{q}-\frac{q_{t}}{t}+\frac{1}{t}\left(\alpha q^{2}+\beta\right)+\gamma q^{3}+\frac{\delta}{q}
$$

where

$$
\alpha=8 \theta_{0}, \quad \beta=4-8 \theta_{\infty}, \quad \gamma=4, \quad \delta=-4 .
$$

Assuming that $\theta_{\infty}$ and $\theta_{0}$ are numerical constants, the function (53) becomes the Hamiltonian of [58) with $p$, $q$ being the canonical variables. Also, if we denote

$$
p_{1}=p, \quad q_{1}=q, \quad p_{2}=\ln k, \quad q_{2}=\theta_{\infty}, \quad p_{3}=\ln a, \quad q_{3}=\theta_{0},
$$

then the whole system [57] becomes Hamiltonian with the same Hamiltonian [53], i.e. with

$$
H=\frac{1}{t}\left(2 p_{1}^{2} q_{1}^{2}+p_{1}\left(2 t-2 t q_{1}^{2}+\left(4 q_{2}-1\right) q_{1}\right)-2 t q_{1}\left(q_{2}+q_{3}\right)+q_{2}^{2}-q_{3}^{2}\right),
$$

and with respect to the symplectic form,

$$
\Omega=d p_{1} \wedge d q_{1}+d p_{2} \wedge d q_{2}+d p_{3} \wedge d q_{3}
$$

The general formulae (3) and (12) transform, in the case of system 48), into the equations,

$$
\omega_{\mathrm{JMU}}=-\operatorname{res}_{z=\infty} \operatorname{Tr}\left(\left(G^{(\infty)}(z)\right)^{-1} \frac{d G^{(\infty)}(z)}{d z} \frac{d \Theta_{\infty}(z)}{d t}\right) d t-\operatorname{res}_{z=0} \operatorname{Tr}\left(\left(G^{(0)}(z)\right)^{-1} \frac{d G^{(0)}(z)}{d z} \frac{d \Theta_{0}(z)}{d t}\right) d t
$$

and

$$
\omega=\operatorname{res}_{z=\infty} \operatorname{Tr}\left(A(z) d G^{(\infty)}(z) G^{(\infty)}(z)^{-1}\right)+\operatorname{res}_{z=0} \operatorname{Tr}\left(A(z) d G^{(0)}(z) G^{(0)}(z)^{-1}\right),
$$

respectively. Substituting the series $G^{(\infty, 0)}(z)$ and the exponentials $\Theta_{\infty, 0}(z)$ from [49] and [51] into [60), we obtain that

$$
\omega_{\mathrm{JMU}}=-\frac{1}{2} \operatorname{Tr}\left(g_{\infty, 1} \sigma_{3}\right) d t-\frac{1}{2} \operatorname{Tr}\left(g_{0,1} \sigma_{3}\right) d t
$$

and using [50] and [52] we arrive at the final formula for $\omega_{\mathrm{JMU}}$,

$$
\omega_{\mathrm{JMU}} \equiv \frac{d \ln \tau}{d t} d t=H d t+\frac{p q}{t} d t-t d t
$$

Note the additional to $H d t$ terms in the right hand side of 62 . Similar substitution of $G^{(\infty, 0)}(z)$ from 49 and 51) into 61 leads us to the formula,

$$
\omega=\operatorname{Tr}\left(A_{-1} d G_{0} G_{0}^{-1}+G_{0}^{-1} A_{-2} G_{0} d g_{0,1}-A_{0} d g_{\infty, 1}\right),
$$


and using again (50), (52) and (54) we arrive at the equation,

$$
\omega=p d q+t d H-\theta_{\infty} \frac{d k}{k}-\theta_{0} \frac{d a}{a}-t d t .
$$

After regrouping the terms we obtain that

$$
\omega=p d q-H d t+d\left(H t-\theta_{\infty} \ln k-\theta_{0} \ln a-\frac{t^{2}}{2}\right)+\ln k d \theta_{\infty}+\ln a d \theta_{0},
$$

or, using the definition (59) of the canonical coordinates,

$$
\omega=p_{1} d q_{1}+p_{2} d q_{2}+p_{3} d q_{3}-H d t+d\left(H t-q_{2} p_{2}-q_{3} p_{3}-\frac{t^{2}}{2}\right) .
$$

Equation (63) proves Conjectures 1 and 2 , with $\gamma=1$, in the case of the $2 \times 2$ system (48) and gives the explicit formula for $G\left(p_{j}, q_{j}, t\right)$,

$$
G\left(p_{1}, p_{2}, p_{3}, q_{1}, q_{2}, q_{3}, t\right)=H t-q_{2} p_{2}-q_{3} p_{3}-\frac{t^{2}}{2} .
$$

The corresponding equation $\underline{18}$ is

$$
\frac{d \ln \tau}{d t}=p \frac{d q}{d t}-H+\frac{d}{d t}\left(H t-\theta_{\infty} \ln k-\theta_{0} \ln a-\frac{t^{2}}{2}\right) .
$$

Remark. One can deduce from (57) that

$$
\frac{p q}{t}=\frac{1}{4} \frac{d}{d t} \ln \frac{a}{k}-\frac{\theta_{0}+\theta_{\infty}}{2 t} .
$$

Combining this with 62) and (64), we arrive at the equation,

$$
H d t=p d q-H d t+d\left(H t+\frac{1-4 \theta_{0}}{4} \ln k-\frac{1+4 \theta_{0}}{4} \ln a+\frac{\theta_{0}+\theta_{\infty}}{2} \ln t\right),
$$

where $d f \equiv d_{t} f=\frac{d f}{d t}$. In other words, although the truncated action, $H d t$, is not in this case exactly the JimboMiwa-Ueno form $\omega_{\text {JMU }}$, it still coincides with the full classical action, up to a total differential. As we will see, this is true in all other examples when accidentally $H d t \neq \omega_{\mathrm{JMU}}$.

\subsection{Painlevé IV}

This time (see again $[\overline{\mathrm{JM}}]$ ), the linear system is the $2 \times 2$ system with one irregular singular point at $z=\infty$ with the Poincaré rank 2 and one Fuchsian point at $z=0$ :

$$
\frac{d \Phi}{d z}=A(z) \Phi, \quad A(z)=\frac{A_{-1}}{z}+A_{0}+A_{1} z,
$$

where

$$
A_{1}=\left(\begin{array}{cc}
1 & 0 \\
0 & -1
\end{array}\right), \quad A_{0}=\left(\begin{array}{cc}
t & k \\
-\frac{q(4 p-q-2 t)+4 \theta_{\infty}}{2 k} & -t
\end{array}\right), \quad A_{-1}=\frac{1}{2}\left(\begin{array}{cc}
\frac{q(4 p-q-2 t)}{2} & -k q \\
\frac{q^{2}(4 p-q-2 t)^{2}-16 \theta_{0}^{2}}{4 k q} & -\frac{q(4 p-q-2 t)}{2}
\end{array}\right) .
$$

The corresponding formal solution at $z=\infty$ is

$$
\Phi_{\text {form }}(z)=\left(I+\frac{g_{1}}{z}+\frac{g_{2}}{z^{2}}+O\left(\frac{1}{z^{3}}\right)\right) e^{\Theta(z)}, \quad \Theta(z)=\sigma_{3}\left(\frac{z^{2}}{2}+t z-\theta_{\infty} \ln z\right)
$$

with

$$
\begin{aligned}
& g_{1}=\frac{1}{2}\left(\begin{array}{cc}
-\frac{2 H+q}{2} & -k \\
-\frac{q(4 p-q-2 t)+4 \theta_{\infty}}{2 k} & \frac{2 H+q}{2}
\end{array}\right), \\
& g_{2}=\frac{1}{8}\left(\begin{array}{cc}
\frac{1}{4}\left((2 H+q+2 t)^{2}-4 t^{2}-8 \theta_{0}^{2}+8 \theta_{\infty}^{2}\right) & -k(2 H-q-4 t) \\
\frac{1}{2 k}\left((2 H-q)\left(q(4 p-q-2 t)+4 \theta_{\infty}+4\right)+8 q\right) & \frac{1}{4}\left((2 H+q+2 t)^{2}-4 t^{2}+8 \theta_{0}^{2}-8 \theta_{\infty}^{2}\right),
\end{array}\right),
\end{aligned}
$$


and

$$
H=2 p^{2} q-\frac{1}{8} q^{3}-\frac{1}{2} t q^{2}+\frac{1}{2}\left(2 \theta_{\infty}-1-t^{2}\right) q+2 \theta_{\infty} t-\frac{2 \theta_{0}^{2}}{q} .
$$

The behavior of the solution of (65) at the (non-resonant, this time) Fuchsian point $z=0$ is described by the equation,

$$
\Phi^{(0)}(z)=G_{0}(I+O(z)) z^{\theta_{0} \sigma_{3}}, \quad z \rightarrow 0,
$$

where $G_{0}$ diagonalizes the matrix $A_{-1}$,

$$
G_{0}^{-1} A_{-1} G_{0}=\theta_{0} \sigma_{3}
$$

and it is chosen in the form,

$$
G_{0}=\frac{1}{2 \sqrt{k q \theta_{0}}}\left(\begin{array}{cc}
-k q & -k q \\
-\frac{q(4 p-q-2 t)-4 \theta_{0}}{2} & -\frac{q(4 p-q-2 t)+4 \theta_{0}}{2}
\end{array}\right) a^{-\frac{\sigma_{3}}{2}} .
$$

The full parameter space,

$$
\mathscr{A}=\left\{p, q, k, a, t, \theta_{0}, \theta_{\infty},\right\},
$$

is again seven dimensional with $t$ being the isomonodromic time and $\theta_{\infty}$ and $\theta_{0}$ serving as the formal monodromy exponents at the respective singular points. The isomonodromicity with respect to $t$ yields the second differential equation for $\Phi(z)$,

$$
\frac{d \Phi}{d t}=B(z) \Phi, \quad B(z)=B_{1} z+B_{0},
$$

where

$$
B_{1}=A_{1}, \quad B_{0}=\left(\begin{array}{cc}
0 & k \\
\frac{q(4 p-q-2 t)+4 \theta_{\infty}}{2 k} & 0
\end{array}\right) .
$$

and the compatibility of (71) and (65) implies,

$$
\begin{aligned}
& \frac{d q}{d t}=4 p q, \\
& \frac{d p}{d t}=-2 p^{2}+\frac{3}{8} q^{2}+q t+\frac{1}{2} t^{2}-\theta_{\infty}+\frac{1}{2}-\frac{2 \theta_{0}^{2}}{q^{2}}, \\
& \frac{d k}{d t}=-(q+2 t) k, \quad \frac{d a}{d t}=\frac{4 \theta_{0}}{q} a, \\
& \frac{d \theta_{\infty}}{d t}=0, \quad \frac{d \theta_{0}}{d t}=0 .
\end{aligned}
$$

As in the previous section, the fourth equation follows from the substitution of [69) into 71].

Similar to the previous cases, the last equations of (72) manifest the time-independence of the formal monodromy exponents, the third and the fourth equations express $k$ and $a$ in terms of $p$ and $q$, while the first and the second equations are equivalent to a Painlevé equation, this time to the fourth Painlevé equation,

$$
q_{t t}=\frac{\left(q_{t}\right)^{2}}{2 q}+\frac{3}{2} q^{3}+4 t q^{2}+2\left(t^{2}-\alpha\right) q+\frac{\beta}{q},
$$

where

$$
\alpha=2 \theta_{\infty}-1, \quad \beta=-8 \theta_{0}^{2} .
$$

Assuming that $\theta_{\infty}$ and $\theta_{0}$ are numerical constants, the function (68) becomes the Hamiltonian of (73) with $p$, $q$ being the canonical variables. Also, if we again denote

$$
p_{1}=p, \quad q_{1}=q, \quad p_{2}=\ln k, \quad q_{2}=\theta_{\infty}, \quad p_{3}=\ln a, \quad q_{3}=\theta_{0},
$$

then the whole system (72) becomes Hamiltonian with the same Hamiltonian (68), i.e. with

$$
H=2 p_{1}^{2} q_{1}-\frac{1}{8} q_{1}^{3}-\frac{1}{2} t q_{1}^{2}+\frac{1}{2}\left(2 q_{2}-1-t^{2}\right) q_{1}+2 q_{2} t-\frac{2 q_{3}^{2}}{q_{1}},
$$


and with respect to the symplectic form,

$$
\Omega=d p_{1} \wedge d q_{1}+d p_{2} \wedge d q_{2}+d p_{3} \wedge d q_{3} .
$$

The general formulae (3) and (12) transform, in the case of system (65), into the equations,

$$
\omega_{\mathrm{JMU}}=-\operatorname{res}_{z=\infty} \operatorname{Tr}\left(\left(G^{(\infty)}(z)\right)^{-1} \frac{d G^{(\infty)}(z)}{d z} \frac{d \Theta_{\infty}(z)}{d t}\right) d t
$$

and

$$
\omega=\operatorname{res}_{z=\infty} \operatorname{Tr}\left(A(z) d G^{(\infty)}(z) G^{(\infty)}(z)^{-1}\right)+\operatorname{res}_{z=0} \operatorname{Tr}\left(A(z) d G^{(0)}(z) G^{(0)}(z)^{-1}\right),
$$

respectively. Substituting the series $G^{(\infty)}(z)$ and the exponentials $\Theta_{\infty}(z)$ from (66) into (75), and using (3.4) we obtain that

$$
\omega_{\mathrm{JMU}} \equiv \frac{d \ln \tau}{d t} d t=H d t+\frac{1}{2} q d t .
$$

Note again the additional to $H d t$ term in the right hand side of 777 . Similar substitution of $G^{(\infty, 0)}(z)$ from 66 , and (69) into (76) followed by the use of (3.4) and (70) leads us to the formulae,

$$
\begin{gathered}
\omega=\operatorname{Tr}\left(A_{-1} d G_{0} G_{0}^{-1}-A_{1} d g_{2}+A_{1} d g_{1} g_{1}-A_{0} d g_{1}\right) \\
=-\frac{1}{2} q d p+\frac{1}{2} p d q+\frac{1}{2} t d H-\frac{1}{2} H d t-\theta_{\infty} \frac{d k}{k}-\theta_{0} \frac{d a}{a}+\theta_{0} d \theta_{0}-\frac{2 \theta_{\infty}-1}{2} d \theta_{\infty} .
\end{gathered}
$$

Regrouping the last equation, we arrive at the final answer for the form $\omega$,

$$
\omega=p d q-H d t+d\left(\frac{H t}{2}-\frac{p q}{2}-\theta_{\infty} \ln k-\theta_{0} \ln a+\frac{\theta_{0}^{2}}{2}+\frac{\theta_{\infty}}{2}-\frac{\theta_{\infty}^{2}}{2}\right)+\ln k d \theta_{\infty}+\ln a d \theta_{0}
$$

or, using the definition (74) of the canonical coordinates,

$$
\omega=p_{1} d q_{1}+p_{2} d q_{2}+p_{3} d q_{3}-H d t+d\left(\frac{H t}{2}-\frac{p_{1} q_{1}}{2}-q_{2} p_{2}-q_{3} p_{3}+\frac{q_{3}^{2}}{2}+\frac{q_{2}}{2}-\frac{q_{2}^{2}}{2}\right) .
$$

Equation (78) proves Conjectures 1 and 2 , with $\gamma=1$, in the case of the $2 \times 2$ system (65) and gives the explicit formula for $G\left(p_{j}, q_{j}, t\right)$,

$$
G\left(p_{1}, p_{2}, p_{3}, q_{1}, q_{2}, q_{3}, t\right)=\frac{H t}{2}-\frac{p_{1} q_{1}}{2}-q_{2} p_{2}-q_{3} p_{3}+\frac{q_{3}^{2}}{2}+\frac{q_{2}}{2}-\frac{q_{2}^{2}}{2} .
$$

The corresponding equation (18) and the formula for the truncated action are

$$
\frac{d \ln \tau}{d t}=p \frac{d q}{d t}-H+\frac{d}{d t}\left(\frac{H t}{2}-\frac{p q}{2}-\theta_{\infty} \ln k-\theta_{0} \ln a\right),
$$

and

$$
H d t=p d q-H d t+d\left(\frac{H t}{2}-\frac{p q}{2}+\frac{1-2 \theta_{\infty}}{2} \ln k-\theta_{0} \ln a+\frac{t^{2}}{2}\right), \quad d \equiv d_{t},
$$

respectively.

\subsection{Painlevé V}

In [JM], the following linear system is associated with the fifth Painlevé equation,

$$
\frac{d \Phi}{d z}=A(z) \Phi, \quad A(z)=A_{2}+\frac{A_{0}}{z}+\frac{A_{1}}{z-1},
$$

where

$$
A_{2}=\frac{t}{2}\left(\begin{array}{cc}
1 & 0 \\
0 & -1
\end{array}\right), \quad A_{0}=\left(\begin{array}{cc}
-p q-\theta_{\infty}-\theta_{1} & k\left(p q+\theta_{\infty}+\theta_{1}-\theta_{0}\right) \\
-\frac{1}{k}\left(p q+\theta_{\infty}+\theta_{1}+\theta_{0}\right) & p q+\theta_{\infty}+\theta_{1}
\end{array}\right),
$$




$$
A_{1}=\left(\begin{array}{cc}
p q+\theta_{1} & -k q\left(p q+2 \theta_{1}\right) \\
\frac{p}{k} & -p q-\theta_{1}
\end{array}\right)
$$

This system has one irregular singular point of Poincaré rank 1 at $z=\infty$ and two Fuchsian singular points $z=0$ and $z=1$. The corresponding formal solution at $z=\infty$ is given by the formulae,

$$
\Phi_{\text {form }}(z)=\left(I+\frac{g_{1}}{z}+O\left(\frac{1}{z^{2}}\right)\right) e^{\Theta(z)}, \quad z \rightarrow \infty, \quad \Theta(z)=\sigma_{3}\left(\frac{t z}{2}-\theta_{\infty} \ln z\right)
$$

with

$$
g_{1}=\left(\begin{array}{cc}
-H & \frac{k\left(p q^{2}-p q+2 \theta_{1} q-\theta_{\infty}-\theta_{1}+\theta_{0}\right)}{t} \\
-\frac{2 p q-2 p+2 \theta_{\infty}+2 \theta_{1}+2 \theta_{0}}{t k} & H
\end{array}\right)
$$

and

$$
\begin{gathered}
H=\frac{p^{2}(q-1)^{2} q}{t}+p\left(\frac{q^{2}}{t}\left(\theta_{0}+3 \theta_{1}+\theta_{\infty}\right)+\frac{q}{t}\left(t-2 \theta_{\infty}-4 \theta_{1}\right)+\frac{1}{t}\left(\theta_{\infty}+\theta_{1}-\theta_{0}\right)\right)+\frac{q 2 \theta_{1}}{t}\left(\theta_{\infty}+\theta_{1}+\theta_{0}\right) \\
+\frac{\theta_{0}^{2}-\theta_{1}^{2}-\theta_{\infty}^{2}+\theta_{1} t-2 \theta_{1} \theta_{\infty}}{t} .
\end{gathered}
$$

The behavior of the solutions of (79) at the (non-resonant) Fuchsian points $z=0$ and $z=1$ are described by the equations,

$$
\Phi^{(0)}(z)=G_{0}(I+O(z)) z^{\theta_{0} \sigma_{3}}, \quad z \rightarrow 0,
$$

and

$$
\Phi^{(1)}(z)=G_{1}(I+O(z-1))(z-1)^{\theta_{1} \sigma_{3}}, \quad z \rightarrow 1,
$$

respectively. The matrices $G_{0}$ and $G_{1}$ diagonalize the matrix coefficients $A_{0}$ and $A_{1}$,

$$
G_{0}^{-1} A_{0} G_{0}=\theta_{0} \sigma_{3}, \quad G_{1}^{-1} A_{1} G_{1}=\theta_{1} \sigma_{3},
$$

and are chosen in the form,

$$
G_{0}=\frac{1}{\sqrt{-4 k \theta_{0}}}\left(\begin{array}{cc}
k\left(2 p q+2 \theta_{\infty}+2 \theta_{1}-2 \theta_{0}\right) & k \\
2 p q+2 \theta_{\infty}+2 \theta_{1}+2 \theta_{0} & 1
\end{array}\right) a^{-\frac{\sigma_{3}}{2}},
$$

and

$$
G_{1}=\frac{1}{\sqrt{2 k \theta_{1}}}\left(\begin{array}{cc}
k\left(p q+2 \theta_{1}\right) & k q \\
p & 1
\end{array}\right) b^{-\frac{\sigma_{3}}{2}} .
$$

The full space

$$
\mathscr{A}=\left\{p, q, k, a, b, t, \theta_{0}, \theta_{1}, \theta_{\infty},\right\} .
$$

is nine dimensional with $t$ being the isomonodromic time and $\theta_{\infty}, \theta_{0}$, and $\theta_{1}$ serving as the formal monodromy exponents at the respective singular points. The isomonodromicity with respect to $t$ yields the second differential equation for $\Phi(z)$,

$$
\frac{d \Phi}{d t}=B(z) \Phi, \quad B(z)=B_{1} z+B_{0},
$$

where

$$
B_{1}=\frac{A_{2}}{t}, \quad B_{0}=\left(\begin{array}{cc}
0 & \frac{k}{t}\left(-p q^{2}+p q-2 \theta_{1} q+\theta_{\infty}+\theta_{1}-\theta_{0}\right) \\
-\frac{1}{t k}\left(p q+\theta_{\infty}-p+\theta_{1}+\theta_{0}\right) & 0
\end{array}\right),
$$


and the compatibility of (87) and (79) implies,

$$
\begin{aligned}
& \frac{d q}{d t}=\frac{2 p q(q-1)^{2}}{t}+\frac{q^{2}}{t}\left(\theta_{0}+3 \theta_{1}+\theta_{\infty}\right)+\frac{q}{t}\left(t-2 \theta_{\infty}-4 \theta_{1}\right)+\frac{1}{t}\left(\theta_{\infty}+\theta_{1}-\theta_{0}\right) \\
& \left.\frac{d p}{d t}=-\frac{p^{2}}{t}\left(3 q^{2}-4 q+1\right)-p\left(\frac{2 q}{t}\left(\theta_{0}+3 \theta_{1}+\theta_{\infty}\right)+\frac{1}{t}\left(t-2 \theta_{\infty}-4 \theta_{1}\right)\right)\right)-\frac{2 \theta_{1}}{t}\left(\theta_{\infty}+\theta_{1}+\theta_{0}\right), \\
& \frac{d k}{d t}=-\frac{k}{t}\left(p q^{2}-2 p q+p+2 \theta_{1} q-2 \theta_{\infty}-2 \theta_{1}\right) \\
& \frac{d a}{d t}=\frac{a}{t}\left(p-p q^{2}-2 \theta_{1} q-2 \theta_{0}\right) \\
& \frac{d b}{d t}=-\frac{b}{t}\left(3 p q^{2}+p-4 p q+2 \theta_{\infty} q+4 \theta_{1} q+2 \theta_{0} q-2 \theta_{\infty}-2 \theta_{1}+t\right) \\
& \frac{d \theta_{\infty}}{d t}=0, \quad \frac{d \theta_{0}}{d t}=0, \quad \frac{d \theta_{1}}{d t}=0 .
\end{aligned}
$$

As before, the equations for $a$ and $b$ follow from the substitution of (83) into (87) and (84) into [87), respectively, and they simply express the functions $a(t)$ and $b(t)$ in terms of $p$ and $q$. The third equation in (88) is also trivial - just an expression of $k$ in terms of $p$ and $q$, and the last three equations are the manifestation of the timeindependence of the formal monodromy exponents. The nontrivial first two equations are equivalent to the fifth Painlevé equation,

$$
q_{t t}=\left(\frac{1}{2 q}+\frac{1}{q-1}\right)\left(q_{t}\right)^{2}-\frac{q_{t}}{t}+\frac{(q-1)^{2}}{t^{2}}\left(\alpha q+\frac{\beta}{q}\right)+\gamma \frac{q}{t}+\delta \frac{q(q+1)}{(q-1)},
$$

where

$$
\alpha=\frac{\left(\theta_{0}-\theta_{1}+\theta_{\infty}\right)^{2}}{2}, \quad \beta=-\frac{\left(\theta_{0}-\theta_{1}-\theta_{\infty}\right)^{2}}{2}, \quad \gamma=\left(1-2 \theta_{0}-2 \theta_{1}\right), \quad \delta=-\frac{1}{2} .
$$

Assuming that $\theta_{\infty}, \theta_{0}$, and $\theta_{1}$ are numerical constants, the function [82] becomes the Hamiltonian of [89] with $p, q$ being the canonical variables. Also, if we denote

$$
p_{1}=p, \quad q_{1}=q, \quad p_{2}=\ln k, \quad q_{2}=\theta_{\infty}, \quad p_{3}=\ln a, \quad q_{3}=\theta_{0}, \quad p_{4}=\ln b, \quad q_{4}=\theta_{1},
$$

then the whole system (88) becomes Hamiltonian with the same Hamiltonian 82, i.e. with

$$
\begin{aligned}
H=\frac{p_{1}^{2}\left(q_{1}-1\right)^{2} q_{1}}{t}+p_{1}\left(\frac { q _ { 1 } ^ { 2 } } { t } \left(q_{3}+3 q_{4}\right.\right. & \left.\left.+q_{2}\right)+\frac{q_{1}}{t}\left(t-2 q_{2}-4 q_{4}\right)+\frac{1}{t}\left(q_{2}+q_{4}-q_{3}\right)\right)+\frac{2 q_{1} q_{4}}{t}\left(q_{2}+q_{4}+q_{3}\right) \\
& +\frac{q_{3}^{2}-q_{4}^{2}-q_{2}^{2}+q_{4} t-2 q_{4} q_{2}}{t},
\end{aligned}
$$

and with respect to the symplectic form,

$$
\Omega=d p_{1} \wedge d q_{1}+d p_{2} \wedge d q_{2}+d p_{3} \wedge d q_{3}+d p_{4} \wedge d q_{4} .
$$

The general formulae (3) and (12) transform, in the case of system (79), into the equations,

$$
\omega_{\mathrm{JMU}}=-\operatorname{res}_{z=\infty} \operatorname{Tr}\left(\left(G^{(\infty)}(z)\right)^{-1} \frac{d G^{(\infty)}(z)}{d z} \frac{d \Theta_{\infty}(z)}{d t}\right) d t
$$

and

$$
\begin{gathered}
\omega=\operatorname{res}_{z=\infty} \operatorname{Tr}\left(A(z) d G^{(\infty)}(z) G^{(\infty)}(z)^{-1}\right)+\operatorname{res}_{z=0} \operatorname{Tr}\left(A(z) d G^{(0)}(z) G^{(0)}(z)^{-1}\right) \\
+\operatorname{res}_{z=1} \operatorname{Tr}\left(A(z) d G^{(1)}(z) G^{(1)}(z)^{-1}\right),
\end{gathered}
$$

respectively. Substituting the series $G^{(\infty)}(z)$ and the exponent $\Theta_{\infty}(z)$ from 80 into 91 and using 81 , we obtain that, similar to the Painlevé II case,

$$
\omega_{\mathrm{JMU}} \equiv \frac{d \ln \tau}{d t} d t=H d t .
$$


Substituting $G^{(\infty, 0,1)}(z)$ from [80, 83, and 84) into (92) and using after that 81, 85], 866 leads us to the formulae,

$$
\begin{gathered}
\omega=\operatorname{Tr}\left(A_{0} d G_{0} G_{0}^{-1}+A_{1} d G_{1} G_{1}^{-1}-A_{2} d g_{1}\right) \\
=p d q+t d H-\theta_{\infty} \frac{d k}{k}-\theta_{0} \frac{d a}{a}-\theta_{1} \frac{d b}{b}+d \theta_{0}+d \theta_{1} .
\end{gathered}
$$

Regrouping the last equation, we arrive at the final answer for the form $\omega$,

$$
\omega=p d q-H d t+d\left(H t-\theta_{\infty} \ln k-\theta_{0} \ln a-\theta_{1} \ln b+\theta_{0}+\theta_{1}\right)+\ln a d \theta_{0}+\ln b d \theta_{1}+\ln k d \theta_{\infty},
$$

or, using the definition 90 of the canonical coordinates,

$$
\omega=p_{1} d q_{1}+p_{2} d q_{2}+p_{3} d q_{3}+p_{4} d q_{4}-H d t+d\left(H t-q_{2} p_{2}-q_{3} p_{3}-q_{4} p_{4}+q_{3}+q_{4}\right) .
$$

Equation (94) proves Conjectures 1 and 2, with $\gamma=1$, in the case of the $2 \times 2$ system (79) and gives the explicit formula for $G\left(p_{j}, q_{j}, t\right)$,

$$
G\left(p_{1}, p_{2}, p_{3}, p_{4}, q_{1}, q_{2}, q_{3}, q_{4}, t\right)=H t-q_{2} p_{2}-q_{3} p_{3}-q_{4} p_{4}+q_{3}+q_{4}
$$

The corresponding equation (18) is

$$
\frac{d \ln \tau}{d t}=p \frac{d q}{d t}-H+\frac{d}{d t}\left(H t-\theta_{\infty} \ln k-\theta_{0} \ln a-\theta_{1} \ln b\right) .
$$

Again, this equation together with 93 make an identity, which, this time, would not be so easy to check directly.

\subsection{Painlevé VI}

Consider the $2 \times 2$ Fuchsian system with 4 regular singularities at $0,1, t$ and $\infty$,

$$
\frac{d \Phi}{d z}=A(z) \Phi, \quad A(z)=\frac{A_{0}}{z}+\frac{A_{t}}{z-t}+\frac{A_{1}}{z-1},
$$

where

$$
A_{0}, A_{1}, A_{t} \in \mathfrak{s l}_{2}(\mathbb{C}), \quad A_{0}+A_{1}+A_{t}=-\theta_{\infty} \sigma_{3} .
$$

Following to $[\mathrm{JM}]$, we introduce the parametrization,

$$
A_{0}=\left(\begin{array}{cc}
x_{0}+\theta_{0} & -u x_{0} \\
\frac{x_{0}+2 \theta_{0}}{u} & -x_{0}-\theta_{0}
\end{array}\right), \quad A_{1}=\left(\begin{array}{cc}
x_{1}+\theta_{1} & -v x_{1} \\
\frac{x_{1}+2 \theta_{1}}{v} & -x_{1}-\theta_{1}
\end{array}\right), \quad A_{t}=\left(\begin{array}{cc}
x_{t}+\theta_{t} & -w x_{t} \\
\frac{x_{t}+2 \theta_{t}}{w} & -x_{t}-\theta_{t}
\end{array}\right) \text {. }
$$

Observe that, $\pm \theta_{0}, \pm \theta_{1}, \pm \theta_{t}$ are the eigenvalues of $A_{0}, A_{1}, A_{t}$, and that the following constraints are satisfied,

$$
\begin{gathered}
x_{0}+\theta_{0}+x_{1}+\theta_{1}+x_{t}+\theta_{t}=-\theta_{\infty}, \\
u x_{0}+v x_{1}+w x_{t}=0, \\
\frac{x_{0}+2 \theta_{0}}{u}+\frac{x_{1}+2 \theta_{1}}{v}+\frac{x_{t}+2 \theta_{t}}{w}=0 .
\end{gathered}
$$

We also introduce the parameters $k$ and $q$ by writing the entry $A_{12}(z)$ of the matrix $A(z)$ as,

$$
A_{1,2}(z)=\frac{k(z-q)}{z(z-1)(z-t)} .
$$

Notice that,

$$
u x_{0}(1+t)+v x_{1}+w x_{t}=k, \quad u x_{0} t=k q .
$$

Finally we put

$$
p=A_{11}(q)=\frac{x_{0}+\theta_{0}}{q}+\frac{x_{1}+\theta_{1}}{q-1}+\frac{x_{t}+\theta_{t}}{q-t} .
$$


Solving equations 97 and 99 with respect to $u, v, w$, we get

$$
u=\frac{k q}{x_{0} t}, \quad v=\frac{k(q-1)}{x_{1}(1-t)}, \quad w=\frac{k(t-q)}{x_{t} t(1-t)} .
$$

Next, we express $x_{1}, x_{t}$ from [96, [100, and then we express $x_{0}$ from (98). The result is

$$
\begin{gathered}
x_{0}=\frac{p^{2} q^{2}(q-1)(q-t)}{t 2 \theta_{\infty}}+\frac{p q(q-1)(q-t)}{t}+\frac{\theta_{\infty} q(q-t-1)}{2 t}+\frac{\theta_{1}^{2}(t-1)}{2 t \theta_{\infty}(q-1)}-\frac{\theta_{t}^{2} t(t-1)}{2 \theta_{\infty}(q-t)} \\
\quad-\frac{\theta_{1}^{2}(1-t)}{2 t \theta_{\infty}}-\frac{\theta_{t}^{2} t(t-1)}{2 t \theta_{\infty}}-\theta_{0}-\frac{\theta_{0}^{2}}{2 \theta_{\infty}}, \\
x_{1}=\frac{p^{2} q(q-1)^{2}(t-q)}{(t-1) 2 \theta_{\infty}}+\frac{p q(q-1)(t-q)}{t-1}+\frac{\theta_{\infty}(q-1)(t-q-1)}{2(t-1)}-\frac{\theta_{0}^{2} t}{2 q \theta_{\infty}(t-1)}+\frac{\theta_{t}^{2} t(t-1)}{2 \theta_{\infty}(q-t)} \\
\quad+\frac{\theta_{0}^{2} t}{2(t-1) \theta_{\infty}}+\frac{\theta_{t}^{2} t(t-1)}{2(t-1) \theta_{\infty}}-\theta_{1}-\frac{\theta_{1}^{2}}{2 \theta_{\infty}}, \\
x_{t}=\frac{p^{2} q(q-1)(t-q)^{2}}{t(t-1) 2 \theta_{\infty}}+\frac{p q(q-1)(q-t)}{t(t-1)}+\frac{\theta_{\infty}(q-t)(q+t-1)}{2 t(t-1)}+\frac{\theta_{0}^{2} t}{2 q \theta_{\infty}(t-1)}-\frac{\theta_{1}^{2}(t-1)}{2 \theta_{\infty} t(q-1)} \\
-\frac{\theta_{0}^{2}}{2(t-1) \theta_{\infty}}+\frac{\theta_{1}^{2}}{2 t \theta_{\infty}}-\theta_{t}-\frac{\theta_{t}^{2}}{2 \theta_{\infty}} .
\end{gathered}
$$

Equations 101 - 102 privide parametrization of the matrices $A_{0}, A_{1}$, and $A_{t}$ by the parameters $q, p, k, \theta_{0}, \theta_{1}, \theta_{t}, \theta_{\infty}$, which will prove to be the Darboux coordinates, and by the parameter $t$ which is the isomonodromic time.

Solutions of 95 have the following behavior at $z=0,1, t$, and $\infty$,

$$
\begin{gathered}
\Phi^{(\infty)}(z)=\left(I+O\left(z^{-1}\right)\right) z^{-\theta_{\infty} \sigma_{3}}, \quad z \rightarrow \infty . \\
\Phi^{(0)}(z)=G_{0}(I+O(z)) z^{\theta_{0} \sigma_{3}}, \quad z \rightarrow 0, \\
\Phi^{(1)}(z)=G_{1}(I+O(z-1))(z-1)^{\theta_{1} \sigma_{3}}, \quad z \rightarrow 1, \\
\Phi^{(t)}(z)=G_{t}\left(I+g_{1}(z-t)+O\left((z-t)^{2}\right)\right)(z-t)^{\theta_{t} \sigma_{3}}, \quad z \rightarrow t,
\end{gathered}
$$

where

$g_{1}=\left(\begin{array}{cc}\frac{H}{2 \theta_{t}}-p \frac{q(q-1)}{2 \theta_{t} t(t-1)}-\frac{\theta_{\infty}(q-t)}{2 \theta_{t} t(t-1)} & \frac{H c}{2 \theta_{t}\left(1-2 \theta_{t}\right)}-p \frac{q(q-1) c}{2 \theta_{t} t(t-1)}-\frac{\theta_{\infty}(q-t) c}{2 \theta_{t} t(t-1)}-\frac{\theta_{t}\left(2 q t-t^{2}-q\right) c}{\left(2 \theta_{t}-1\right) t(t-1)(q-t)} \\ \frac{H}{2 \theta_{t}\left(1+2 \theta_{t}\right) c}+p \frac{q(q-1)}{2 \theta_{t} t(t-1) c}+\frac{\theta_{\infty}(q-t)}{2 \theta_{t} t(t-1) c}-\frac{\theta_{t}\left(2 q t-t^{2}-q\right)}{\left(2 \theta_{t}+1\right) t(t-1)(q-t) c} & -\frac{H}{2 \theta_{t}}+p \frac{q(q-1)}{2 \theta_{t} t(t-1)}+\frac{\theta_{\infty}(q-t)}{2 \theta_{t} t(t-1)}\end{array}\right)$

and

$$
H=p^{2} \frac{q(q-1)(q-t)}{t(t-1)}+p \frac{q(q-1)}{t(t-1)}+\frac{\theta_{\infty}\left(1-\theta_{\infty}\right)(q-t)}{t(t-1)}+\frac{\theta_{0}^{2}(q-t)}{q t(t-1)}-\frac{\theta_{1}^{2}(q-t)}{(q-1) t(t-1)}+\frac{\theta_{t}^{2}\left(t^{2}-q(2 t-1)\right)}{(q-t) t(t-1)} .
$$

The matrices $G_{0}, G_{1}$, and $G_{t}$ diagonalize the matrix residues $A_{0}, A_{1}$, and $A_{t}$,

$$
G_{0}^{-1} A_{0} G_{0}=\theta_{0} \sigma_{3}, \quad G_{1}^{-1} A_{1} G_{1}=\theta_{1} \sigma_{3}, \quad G_{t}^{-1} A_{t} G_{t}=\theta_{t} \sigma_{3} .
$$

and they are chosen in the form,

$$
\begin{gathered}
G_{0}=\sqrt{\frac{k q}{t}}\left(\begin{array}{cc}
1 & 1 \\
\frac{1}{u} & \frac{x_{0}+2 \theta_{0}}{u x_{0}}
\end{array}\right) a^{-\frac{\sigma_{3}}{2}}, \\
G_{1}=\sqrt{\frac{k(q-1)}{1-t}}\left(\begin{array}{cc}
1 & 1 \\
\frac{1}{v} & \frac{x_{1}+2 \theta_{1}}{v x_{1}}
\end{array}\right) b^{-\frac{\sigma_{3}}{2}}, \\
G_{t}=\sqrt{\frac{k(t-q)}{t(1-t)}}\left(\begin{array}{cc}
1 & 1 \\
\frac{1}{w} & \frac{x_{t}+2 \theta_{t}}{w x_{t}}
\end{array}\right) c^{-\frac{\sigma_{3}}{2}} .
\end{gathered}
$$


The whole parameter space $\mathscr{A}$ has dimension 11 ,

$$
\mathscr{A}=\left\{p, q, k, a, b, c, t, \theta_{0}, \theta_{1}, \theta_{t}, \theta_{\infty}\right\} .
$$

The isomonodromicity with respect to $t$ yields the second differential equation for $\Phi(z)$,

$$
\frac{d \Phi}{d t}=-\frac{A_{t}}{z-t} \Phi
$$

and the equations

$$
\frac{d G_{0}}{d t}=\frac{A_{t}}{t} G_{0}, \quad \frac{d G_{1}}{d t}=\frac{A_{t}}{t-1} G_{1}, \quad \frac{d G_{t}}{d t}=\left(\frac{A_{0}}{t}+\frac{A_{1}}{t-1}\right) G_{t},
$$

for the gauge matrices $G_{0}, G_{1}, G_{t}$. The compatibility of (109) and (95) together with the equations (110) imply the following dynamical system on (108),

$$
\begin{gathered}
\frac{d q}{d t}=\frac{2 p q(q-1)(q-t)}{t(t-1)}+\frac{q(q-1)}{t(t-1)}, \\
\frac{d p}{d t}=\frac{1}{4 t(t-1)}\left(4 p^{2}\left(2 t q-3 q^{2}-t+2 q\right)+4 p(1-2 q)+4 \theta_{\infty}\left(\theta_{\infty}-1\right)\right)-\frac{\theta_{0}^{2}}{q^{2}(t-1)}+\frac{\theta_{1}^{2}}{t(q-1)^{2}}-\frac{\theta_{t}^{2}}{(q-t)^{2}} \\
\frac{d k}{d t}=\frac{k\left(2 \theta_{\infty}-1\right)(q-t)}{t(t-1)}, \\
\frac{d a}{d t}=-\frac{2 \theta_{0}(q-t) a}{q t(t-1)}, \quad \frac{d b}{d t}=\frac{2 \theta_{1}(q-t) b}{t(t-1)(q-1)}, \quad \frac{d c}{d t}=\frac{2 \theta_{t}\left(q(2 t-1)-t^{2}\right) c}{(q-t) t(t-1)}, \\
\frac{d \theta_{0}}{d t}=\frac{d \theta_{1}}{d t}=\frac{d \theta_{t}}{d t}=\frac{d \theta_{\infty}}{d t}=0 .
\end{gathered}
$$

As before, the only non-trivial equations are the first two, and they are equivalent to the sixth Painlevé equation for the function $q(t)$,

$$
\begin{aligned}
\frac{d^{2} q}{d t^{2}} & =\frac{1}{2}\left(\frac{1}{q}+\frac{1}{q-1}+\frac{1}{q-t}\right)\left(\frac{d q}{d t}\right)^{2}-\left(\frac{1}{t}+\frac{1}{t-1}+\frac{1}{q-t}\right) \frac{d q}{d t} \\
& +\frac{q(q-1)(q-t)}{t^{2}(t-1)^{2}}\left(\alpha+\beta \frac{t}{q^{2}}+\gamma \frac{t-1}{(q-1)^{2}}+\delta \frac{t(t-1)}{(q-t)^{2}}\right),
\end{aligned}
$$

where

$$
\alpha=\frac{\left(2 \theta_{\infty}-1\right)^{2}}{2}, \quad \beta=-2 \theta_{0}^{2}, \quad \gamma=2 \theta_{1}^{2}, \quad \delta=\frac{1-4 \theta_{t}^{2}}{2} .
$$

Assuming that $\theta_{j}, j=0,1, t, \infty$ are numerical constants, the function (104) becomes the Hamiltonian of [113 with $p, q$ being the canonical variables. Also, if we denote

$$
\begin{gathered}
p_{1}=p, \quad q_{1}=q, \quad p_{2}=\ln k, \quad q_{2}=\theta_{\infty}, \quad p_{3}=\ln a, \quad q_{3}=\theta_{0}, \\
p_{4}=\ln b, \quad q_{4}=\theta_{1}, \quad p_{5}=\ln c, \quad q_{5}=\theta_{t} .
\end{gathered}
$$

then the whole system (111)-112) becomes Hamiltonian with the same Hamiltonian [104, that is with,

$$
\begin{gathered}
H=p_{1}^{2} \frac{q_{1}\left(q_{1}-1\right)\left(q_{1}-t\right)}{t(t-1)}+p_{1} \frac{q_{1}\left(q_{1}-1\right)}{t(t-1)}+\frac{q_{2}\left(1-q_{2}\right)\left(q_{1}-t\right)}{t(t-1)}+\frac{q_{3}^{2}\left(q_{1}-t\right)}{q_{1} t(t-1)}-\frac{q_{4}^{2}\left(q_{1}-t\right)}{\left(q_{1}-1\right) t(t-1)} \\
+\frac{q_{5}^{2}\left(t^{2}-q_{1}(2 t-1)\right)}{\left(q_{1}-t\right) t(t-1)},
\end{gathered}
$$

and with respect to the symplectic form,

$$
\Omega=\sum_{j=1}^{5} d p_{j} \wedge d q_{j}
$$


The general formulae (3) and (12) transform, in the case of system [95), into the equations,

$$
\omega_{\mathrm{JMU}}=-\operatorname{res}_{z=t} \operatorname{Tr}\left(\left(G^{(t)}(z)\right)^{-1} \frac{d G^{(t)}(z)}{d z} \frac{d \Theta_{t}(z)}{d t}\right) d t
$$

and

$$
\begin{gathered}
\omega=\operatorname{res}_{z=\infty} \operatorname{Tr}\left(A(z) d G^{(\infty)}(z) G^{(\infty)}(z)^{-1}\right)+\operatorname{res}_{z=0} \operatorname{Tr}\left(A(z) d G^{(0)}(z) G^{(0)}(z)^{-1}\right) \\
\operatorname{res}_{z=1} \operatorname{Tr}\left(A(z) d G^{(1)}(z) G^{(1)}(z)^{-1}\right)+\operatorname{res}_{z=t} \operatorname{Tr}\left(A(z) d G^{(t)}(z) G^{(t)}(z)^{-1}\right) .
\end{gathered}
$$

From [115] it follows that

$$
\omega_{\mathrm{JMU}}=\theta_{t} \operatorname{Tr}\left(g_{1} \sigma_{3}\right),
$$

and taking into account 103, we obtain that,

$$
\omega_{\mathrm{JMU}} \equiv \frac{d \ln \tau}{d t} d t=H d t-p \frac{q(q-1)}{t(t-1)} d t-\frac{\theta_{\infty}(q-t)}{t(t-1)} d t .
$$

Similarly, (116) reduces to the equation,

$$
\omega=\operatorname{Tr}\left(\mathrm{G}_{0}^{-1} \mathrm{~A}_{0} d \mathrm{G}_{0}+\mathrm{G}_{1}^{-1} \mathrm{~A}_{1} \mathrm{dG}_{1}+\mathrm{G}_{\mathrm{t}}^{-1} \mathrm{~A}_{\mathrm{t}} \mathrm{dG}_{\mathrm{t}}-\mathrm{A}_{\mathrm{t}} \mathrm{G}_{\mathrm{t}} \mathrm{g}_{1} \mathrm{G}_{\mathrm{t}}^{-1} \mathrm{dt}\right),
$$

which after using (105)-(107) and simplifying yields the formula

$$
\omega=p d q-H d t-\theta_{\infty} \frac{d k}{k}-\theta_{0} \frac{d a}{a}-\theta_{1} \frac{d b}{b}-\theta_{t} \frac{d c}{c}+d \theta_{\infty} .
$$

This, in turn, can be rewritten as

$$
\omega=p d q-H d t+d\left(\theta_{\infty}-\theta_{0} \ln a-\theta_{1} \ln b-\theta_{t} \ln c-\theta_{\infty} \ln k\right)+\ln k d \theta_{\infty}+\ln a d \theta_{0}+\ln b d \theta_{1}+\ln c d \theta_{t} .
$$

or, remembering the definitions (114) of the canonical coordinates,

$$
\omega=\sum_{j=1}^{5} p d q-H d t+d\left(q_{2}-q_{3} p_{3}-q_{4} p_{4}-q_{5} p_{5}-q_{2} p_{2}\right) .
$$

Equation (117) proves Conjectures 1 and 2, with $\gamma=1$, in the case of the $2 \times 2$ system (95) and gives the explicit formula for $G\left(p_{j}, q_{j}, t\right)$,

$$
G\left(p_{1}, p_{2}, p_{3}, p_{4}, p_{5}, q_{1}, q_{2}, q_{3}, q_{4}, q_{5}, t\right)=q_{2}-q_{3} p_{3}-q_{4} p_{4}-q_{5} p_{5}-q_{2} p_{2} .
$$

The corresponding equation (18) and the truncated action are

$$
\frac{d \ln \tau}{d t}=p \frac{d q}{d t}-H-\frac{d}{d t}\left(\theta_{0} \ln a+\theta_{1} \ln b+\theta_{t} \ln c+\theta_{\infty} \ln k\right),
$$

and

$$
H d t=p d q-H d t+d\left(\frac{1}{2} \ln \left(\frac{k(q-t)}{t(t-1)}\right)-\theta_{0} \ln a-\theta_{1} \ln b-\theta_{t} \ln c-\theta_{\infty} \ln k\right), \quad d \equiv d_{t},
$$

respectively.

\subsection{Schlesinger system}

This section reproduces the result of Mal2 (Subsection 5.6, Remark 5.5). Once again, we are grateful to M. Mazzocco for informing us about this part of Malgrange's work.

Consider the Fuchsian system

$$
\frac{d \Phi}{d z}=A(z) \Phi(z), \quad A(z)=\sum_{v=1}^{n} \frac{A_{v}}{z-a_{v}}, \quad A_{v} \in \mathfrak{s l}_{N}(\mathbb{C}) .
$$


We assume that all matrix coefficients $A_{v}$ are diagonalizable

$$
A_{v}=G_{v} \Theta_{v} G_{v}^{-1} ; \quad \Theta_{v}=\operatorname{diag}\left\{\theta_{v, 1}, \ldots \theta_{v, N}\right\},
$$

and that their eigenvalues are distinct and non-resonant:

$$
\theta_{v, \alpha} \neq \theta_{v, \beta} \quad \bmod \mathbb{Z} .
$$

We also assume that the residue of $A(z)$ at $z=\infty$ is diagonal, i.e.

$$
A_{\infty}=-\sum_{v=1}^{n} A_{v}=\Theta_{\infty}
$$

Solutions of (118) have the following behavior at the singular points

$$
\begin{gathered}
\Phi^{(\infty)}(z)=\left(I+O\left(z^{-1}\right)\right) z^{-\Theta_{\infty}}, \quad z \rightarrow \infty, \\
\Phi^{(v)}(z)=G_{v}\left(I+g_{v, 1}\left(z-a_{v}\right)+O\left(\left(z-a_{v}\right)^{2}\right)\right)\left(z-a_{v}\right)^{\Theta_{v}} C_{v}, \quad z \rightarrow a_{v} .
\end{gathered}
$$

The isomonodromic times are now positions of the singular points $a_{v}$. The isomonodromic deformations with respect to these times yields the equation,

$$
\frac{d \Phi}{d a_{v}}=B_{v}(z) \Phi(z), \quad B_{v}(z)=-\frac{A_{v}}{z-a_{v}} .
$$

The compatibility conditions give the Schlesinger system

$$
\frac{d A_{\mu}}{d a_{v}}=\frac{\left[A_{\mu}, A_{v}\right]}{a_{\mu}-a_{v}}, \quad \mu \neq v, \quad \frac{d A_{v}}{d a_{v}}=-\sum_{\mu \neq v} \frac{\left[A_{\mu}, A_{v}\right]}{a_{\mu}-a_{v}},
$$

and also the equations (cf. equations (110),

$$
\frac{d G_{\mu}}{d a_{v}}=\frac{A_{v}}{a_{v}-a_{\mu}} G_{\mu}, \quad \mu \neq v, \quad \frac{d G_{v}}{d a_{v}}=-\sum_{\mu \neq v} \frac{A_{\mu}}{a_{\mu}-a_{v}} G_{v} .
$$

Following [JMMS] we introduce matrix Darboux coordinates

$$
Q_{v}=G_{v} \Theta_{v}, \quad P_{v}=G_{v}^{-1}
$$

and Hamiltonians

$$
H_{v}=\sum_{\mu \neq v}^{n} \frac{\operatorname{Tr}\left(Q_{\mu} P_{\mu} Q_{v} P_{v}\right)}{a_{v}-a_{\mu}} .
$$

Notice that $A_{v}=Q_{v} P_{v}$. Then, as it is shown in Mal2, the isomonodromic equations (119)-120 are equivalent to the Hamiltonian system,

$$
\frac{d P_{\mu, j k}}{d a_{v}}=-\frac{\partial H_{v}}{\partial Q_{\mu, k j}}, \quad \frac{d Q_{\mu, j k}}{d a_{v}}=\frac{\partial H_{v}}{\partial P_{\mu, k j}} .
$$

Moreover, by a rather staightforward calculation, one can show that the general formulae (3) and (12) in the case of the Fuchsian system (118) produce the following expressions of the forms $\omega_{\text {JMU }}$ and $\omega$,

$$
\omega_{\mathrm{JMU}}=\sum_{v=1}^{n} H_{v} d a_{v}
$$

and

$$
\omega=\sum_{v=1}^{n} \operatorname{Tr}\left(\mathrm{P}_{v} \mathrm{dQ}_{v}\right)-H_{v} d a_{v} .
$$

In other words, we have validity of Conjectures 1 and 2 , with $\gamma=1$, in the case of the Fuchsian system 118 . Moreover, the form $\omega$ just coincides with $\omega_{\text {cla }}$. 


\section{Appendix.}

\subsection{The proof of Lemma1}

The proof of Lemma \is rather short. Indeed, noticing that

$$
\left(G^{(v)}\right)^{-1} \frac{d G^{(v)}}{d z}=\left(G^{(v)}\right)^{-1} A G^{(v)}-\frac{d \Theta_{v}}{d z}
$$

and plugging this into the right have side of (3), we have,

$$
\omega_{\mathrm{JMU}}=-\sum_{k=1}^{L} \sum_{v=1, \ldots, n, \infty} \operatorname{res}_{z=a_{v}} \operatorname{Tr}\left(\left(G^{(v)}\right)^{-1} A G^{(v)} \frac{\partial \Theta_{v}}{\partial t_{k}}\right) d t_{k}+\sum_{k=1}^{L} \sum_{v=1, \ldots, n, \infty} \operatorname{res}_{z=a_{v}} \operatorname{Tr}\left(\frac{d \Theta_{v}}{d z} \frac{\partial \Theta_{v}}{\partial t_{k}}\right) d t_{k} .
$$

The expression $\frac{d \Theta_{v}}{d z} \frac{\partial \Theta_{v}}{\partial t_{k}}$ has poles of order at least 2, so it does not have residues and hence the second sum in (122) vanishes. We also have,

$$
\frac{\partial \Theta_{v}}{\partial t_{k}}=\left(G^{(v)}\right)^{-1} B_{k} G^{(v)}-\left(G^{(v)}\right)^{-1} \frac{\partial G^{(v)}}{\partial t_{k}} .
$$

Substituting (123) into [122) we transform it to the equation,

$$
\omega_{\mathrm{JMU}}=-\sum_{k=1}^{L} \sum_{v=1, \ldots, n, \infty} \operatorname{res}_{z=a_{v}} \operatorname{Tr}\left(A B_{k}\right) d t_{k}+\sum_{k=1}^{L} \sum_{v=1, \ldots, n, \infty} \operatorname{res}_{z=a_{v}} \operatorname{Tr}\left(A \frac{\partial G^{(v)}}{\partial t_{k}}\left(G^{(v)}\right)^{-1}\right) d t_{k} .
$$

The function $A B_{k}$ is rational, therefore sum of its residues is zero. So we get (10).

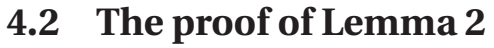

Denote

$$
I=\sum_{k=1}^{L} \sum_{j=1}^{d} \sum_{v=1, \ldots, n, \infty} \operatorname{res}_{z=a_{v}} \operatorname{Tr}\left(\frac{\partial}{\partial m_{j}}\left(A \frac{\partial G^{(v)}}{\partial t_{k}}\left(G^{(v)}\right)^{-1}\right)-\frac{\partial}{\partial t_{k}}\left(A \frac{\partial G^{(v)}}{\partial m_{j}}\left(G^{(v)}\right)^{-1}\right)\right) .
$$

We have

$$
\begin{aligned}
I=\sum_{k=1}^{L} \sum_{j=1}^{d} \sum_{v=1, \ldots, n, \infty} \operatorname{res}_{z=a_{v}} \operatorname{Tr}\left(\frac{\partial A}{\partial m_{j}} \frac{\partial G^{(v)}}{\partial t_{k}}\left(G^{(v)}\right)^{-1}-A \frac{\partial G^{(v)}}{\partial t_{k}}\left(G^{(v)}\right)^{-1} \frac{\partial G^{(v)}}{\partial m_{j}}\left(G^{(v)}\right)^{-1}-\frac{\partial A}{\partial t_{k}} \frac{\partial G^{(v)}}{\partial m_{j}}\left(G^{(v)}\right)^{-1}\right) \\
+\sum_{k=1}^{L} \sum_{j=1}^{d} \sum_{v=1, \ldots, n, \infty} \operatorname{res}_{z=a_{v}} \operatorname{Tr}\left(A \frac{\partial G^{(v)}}{\partial m_{j}}\left(G^{(v)}\right)^{-1} \frac{\partial G^{(v)}}{\partial t_{k}}\left(G^{(v)}\right)^{-1}\right) .
\end{aligned}
$$

We use the formula [123) to get rid of $\frac{\partial G^{(v)}}{\partial t_{k}}$ and equation [6] to replace $\frac{\partial A}{\partial t_{k}}$. Omitting terms with zero residue and after some cancellations we have

$$
\begin{aligned}
I=\sum_{k=1}^{L} \sum_{j=1}^{d} \sum_{v=1, \ldots, n, \infty} \operatorname{res}_{z=a_{v}} \operatorname{Tr} & \left(-\frac{\partial A}{\partial m_{j}} G^{(v)} \frac{\partial \Theta_{v}}{\partial t_{k}}\left(G^{(v)}\right)^{-1}+A G^{(v)} \frac{d \partial \Theta_{v}}{\partial t_{k}}\left(G^{(v)}\right)^{-1} \frac{\partial G^{(v)}}{\partial m_{j}}\left(G^{(v)}\right)^{-1}-\frac{d B_{k}}{d z} \frac{\partial G^{(v)}}{\partial m_{j}}\left(G^{(v)}\right)^{-1}\right) \\
& +\sum_{k=1}^{L} \sum_{j=1}^{d} \sum_{v=1, \ldots, n, \infty} \operatorname{res}_{z=a_{v}} \operatorname{Tr}\left(-A \frac{d \partial G^{(v)}}{\partial m_{j}} \frac{\partial \Theta_{v}}{\partial t_{k}}\left(G^{(v)}\right)^{-1}\right) .
\end{aligned}
$$

We replace $B_{k}$ using again formula (123). After that, we notice that the residue of the derivative with respect to $\mathrm{z}$ of formal series is zero. Therefore we can "integrate by parts", moving the derivative from one term to another. We do that with the term, where we replaced $B_{k}$. Using [121], we have

$$
I=\sum_{k=1}^{L} \sum_{j=1}^{d} \sum_{v=1, \ldots, n, \infty} \operatorname{res}_{z=a_{v}} \operatorname{Tr}\left(-\frac{\partial A}{\partial m_{j}} G^{(v)} \frac{\partial \Theta_{v}}{\partial t_{k}}\left(G^{(v)}\right)^{-1}+A G^{(v)} \frac{\partial \Theta_{v}}{\partial t_{k}}\left(G^{(v)}\right)^{-1} \frac{d G^{(v)}}{\partial m_{j}}\left(G^{(v)}\right)^{-1}-A \frac{\partial G^{(v)}}{\partial m_{j}} \frac{\partial \Theta_{v}}{\partial t_{k}}\left(G^{(v)}\right)^{-1}\right)
$$




$$
\begin{gathered}
+\sum_{k=1}^{L} \sum_{j=1}^{d} \sum_{v=1, \ldots, n, \infty} \operatorname{res}_{z=a_{v}} \operatorname{Tr}\left(-G^{(v)} \frac{\partial \Theta_{v}}{\partial t_{k}}\left(G^{(v)}\right)^{-1} \frac{\partial G^{(v)}}{\partial m_{j}}\left(G^{(v)}\right)^{-1} A+\frac{\partial \Theta_{v}}{\partial t_{k}}\left(G^{(v)}\right)^{-1} \frac{\partial G^{(v)}}{\partial m_{j}} \frac{d \Theta_{v}}{d z}\right) \\
+\sum_{k=1}^{L} \sum_{j=1}^{d} \sum_{v=1, \ldots, n, \infty} \operatorname{res}_{z=a_{v}} \operatorname{Tr}\left(G^{(v)} \frac{\partial \Theta_{v}}{\partial t_{k}}\left(G^{(v)}\right)^{-1} \frac{\partial^{2} G^{(v)}}{\partial z \partial m_{j}}\left(G^{(v)}\right)^{-1}\right) .
\end{gathered}
$$

Finally using [121] one more time we get $I=0$.

Acknowledgements. In addition to M. Mazzocco, we would also like to thank P. Boalch and O. Lisovyy for many very useful discussions and comments. The present work was supported by the NSF Grant DMS-1700261 and Russian Science Foundation grant No.17-11-01126.

\section{References}

[B] M. Babich, On canonical parametrization of the phase spaces of equations of isomonodromic deformations of Fuchsian systems of dimension $2 \times 2$. Derivation of the Painlevé VI equation, UMN, 64:1 (2009), 51-134;(Russian). English translation in Russian Math. Surveys, 64:1 (2009), 45-127.

[Ber] M. Bertola, The dependence on the monodromy data of the isomonodromic tau function, Comm. Math. Phys. 294, (2010), 539-579; arXiv: 0902.4716 [nlin.SI].

[Boal] P. Boalch, Symplectic Manifolds and Isomonodromic Deformations, Advances in Mathematics 163, 137205 (2001).

[Boal2] P. Boalch, Simply-laced isomonodromy systems, Publications mathématiques de l'IHÉS, 116, 1-68 (2012).

[BK] E. Brézin, V. A. Kazakov, Exactly solvable field theories of closed strings, Phys. Letts. B236, (1990), 144-150.

[BIP] T. Bothner, A. Its, A. Prokhorov, On the analysis of incomplete spectra in random matrix theory through an extension of the Jimbo-Miwa-Ueno differential, arXiv:1708.06480 v1 [math-ph].

[BBDI] J. Baik, R. Buckingham, J. DiFranco, A. Its, Total integrals of global solutions to Painlevé II, Nonlinearity 22, (2009), 1021-1061; arXiv:0810.2586 [math.CA].

[DS] M. Douglas, S. Shenker, Strings in less than one dimension, Nucl. Phys. B335, (1990), 635-654.

[FN] H. Flaschka, A. C. Newell, Monodromy- and spectrum-preserving deformations I, Comm. Math. Phys. 76, (1980) 65-116.

[FIKN] A. S. Fokas, A. R. Its, A. A. Kapaev, V. Yu. Novokshenov, Painlevé transcendents: the Riemann-Hilbert approach, Mathematical Surveys and Monographs 128, AMS, Providence, RI, (2006).

[GL] P. Gavrylenko, O. Lisovyy, Fredholm determinant and Nekrasov sum representations of isomonodromic tau functions, (2016); arXiv:1608.00958 [math-ph].

[GM] D. Gross, A. Migdal, A nonperturbative treatment of two-dimensional quantum gravity, Nucl. Phys. B340, (1990), 333-365.

[H1] J. Harnad, M. A. Wisse, Loop algebra moment maps and Hamiltonian models for the Painlevé transcendants, Field's Inst. Commun. 7 (1993), 155?169.

[H2] J. Harnad, M. A. Wisse, Moment Maps to Loop Algebras, Classical R-Matrix and Integrable Systems, "Quantum Groups Integrable Models and Statistical Systems", World Scientific, Singapore (1993), 105-117.

[IP] A. Its, A. Prokhorov, Connection problem for the tau-function of the sine-Gordon reduction of Painlevé-III equation via the Riemann-Hilbert approach, IMRN 22, (2016) 6856-?6883.

[ILP] A. Its, O. Lisovyy, A. Prokhorov, Monodromy dependence and connection formulae for isomonodromic tau functions, arXiv:1604.03082 v1 [math-ph], accepted to Duke Mathematical Journal. 
[ILST] N. Iorgov, O. Lisovyy, A. Shchechkin, Yu. Tykhyy, Painlevé functions and conformal blocks, Constr. Approx. 39, (2014), 255-272.

[ILT13] N. Iorgov, O. Lisovyy, Yu. Tykhyy, Painlevé VI connection problem and monodromy of $c=1$ conformal blocks, JHEP 12, (2013), 029;=arXiv:1308.4092v1 [hep-th].

[ILT14] A. Its, O. Lisovyy, Yu. Tykhyy, Connection problem for the sine-Gordon/Painlevé III tau function and irregular conformal blocks, Int. Math. Res. Not., (2014), rnu209; arXiv: 1403.1235 [math-ph].

Physica D2, (1981), 306-352.

[JMU] M. Jimbo, T. Miwa, K. Ueno, Monodromy preserving deformation of linear ordinary differential equations with rational coefficients. I, Physica D2, (1981), 306-352.

[JM] M. Jimbo, T. Miwa Monodromy preserving deformation of linear ordinary differential equations with rational coefficients. II, Physica D2, (1981), 407-448.

[JMMS] M. Jimbo, T. Miwa, Y. Môri, M. Sato, Density matrix of an impenetrable Bose gas and the fifth Painlevé transcendent, Physica 1D, (1980), 80-158.

[Kri] I. Krichever, Isomonodromy equations on algebraic curves, cannonical transformations and Whitham equations, Moscow Math. J. 2, (2002), 717 - 752; arXiv:hep-th/0112096.

[LR] O. Lisovyy, J. Roussillon On the connection problem for Painlevé I, J. Phys. A: Math. Theor. 50 (2017) 255202

[Mal] B. Malgrange, Sur les déformations isomonodromiques, I. Singularités régulières, in "Mathematics and Physics”, (Paris, 1979/1982); Prog. Math. 37, Birkhäuser, Boston, MA, (1983), 401-426.

[Mal2] B. Malgrange, Déformations isomonodromiques, forme de Liouville, fonction $\tau$, Annales de l'institut Fourier, Volume 54, (2004) no. 5, 1371-1392.

[Miw] T. Miwa, Painlevé property of monodromy preserving deformation equations and the analyticity of $\tau$ functions, Publ. Res. Inst. Math. Sci. 17, (1981), 703-712.

[O] K. Okamoto, Polynomial Hamiltonians associated with Painlevé equations, I, Proc. Japan Acad. Ser. A Math. Sci. Volume 56, Number 6 (1980), 264-268.

[Tr] C. A. Tracy, Asymptotics of the $\tau$-function arising in the two-dimensional Ising model, Comm. Math. Phys. 142, (1991), 297-311.

[TW1] C. A. Tracy, H. Widom, Level-spacing distributions and the Airy kernel, Comm. Math. Phys. 159, (1994), 151-174; hep-th/9211141.

[TW2] C. A. Tracy, H. Widom, Fredholm determinants, differential equations and matrix models, Comm. Math. Phys. 163, (1994), 33-72; hep-th/9306042.

[Was] W. Wasow, Asymptotic expansions for ordinary differential equations, Dover, New York, (2002). 